\title{
Shenlian (SL) Decoction, a Traditional Chinese Medicine Compound, May Ameliorate Blood Glucose via Mediating the Gut Microbiota in $\mathbf{d b} / \mathbf{d b}$ Mice
}

\author{
Rui-xi Sun $\mathbb{D}^{1},{ }^{1}$ Wei-jun Huang $\mathbb{D}^{\circ},{ }^{2}$ Yao Xiao ${ }^{D},{ }^{3}$ Dou-dou Wang, ${ }^{4}$ Guo-hua Mu ${ }^{D},{ }^{4}$ \\ He Nan $\mathbb{D}^{4},{ }^{4}$ Bo-ran Ni $\mathbb{D}^{4},{ }^{4}$ Xiao-qiang Huang $\mathbb{D}^{4},{ }^{4}$ Hsuan-chuan Wang $\mathbb{D}^{4},{ }^{4}$ Yi-fan Liu $\mathbb{D}^{4},{ }^{4}$ \\ Qiang Fu ${ }^{\mathbb{D}},{ }^{4}$ and Jin-xi Zhao $\mathbb{i}^{4}$ \\ ${ }^{1}$ Beijing University of Chinese Medicine, Beijing, China \\ ${ }^{2}$ Key Laboratory of Chinese Internal Medicine of Ministry of Education and Beijing, Dongzhimen Hospital Affiliated to Beijing \\ University of Chinese Medicine, Beijing University of Chinese Medicine, Beijing, China \\ ${ }^{3}$ Nephropathy Department, Beijing University of Chinese Medicine Third Affiliated Hospital, Beijing University of Chinese Medicine, \\ Beijing, China \\ ${ }^{4}$ Section II of Endocrinology \& Nephropathy Department, Dongzhimen Hospital Affiliated to Beijing University of Chinese Medicine, \\ Beijing University of Chinese Medicine, Beijing, China
}

Correspondence should be addressed to Qiang Fu; 13693332059@163.com and Jin-xi Zhao; zhaojinximd@126.com

Received 7 September 2021; Revised 17 December 2021; Accepted 6 January 2022; Published 9 February 2022

Academic Editor: Ike S. Okosun

Copyright (c) 2022 Rui-xi Sun et al. This is an open access article distributed under the Creative Commons Attribution License, which permits unrestricted use, distribution, and reproduction in any medium, provided the original work is properly cited.

Shenlian (SL) decoction is a herbal formula composed of Coptis and ginseng, of which berberine and ginsenoside are the main constituents. Even though SL decoction is widely used in treating diabetes in China, the mechanism of its antidiabetes function still needs further study. Gut microbiota disorder is one of the important factors that cause diabetes. To explore the effect of SL decoction on intestinal microbiota, gut microbiota of mice was analyzed by sequencing the gut bacterial 16S rRNA V3+V4 region and metagenomics. In this study, results demonstrated that SL decoction had a better hypoglycemic effect and $\beta$ cell protection effect than either ginseng or Coptis chinensis. Alpha diversity analysis showed that all interventions with ginseng, Coptis, and SL decoction could reverse the increased diversity and richness of gut microbiota in $\mathrm{db} / \mathrm{db}$ mice. PCoA analysis showed oral SL decoction significantly alters gut microbiota composition in $\mathrm{db} / \mathrm{db}$ mice. 395 OTUs showed significant differences after SL treatment, of which 37 OTUs enriched by SL decoction showed a significant negative correlation with FBG, and 204 OTUs decreased by SL decoction showed a significant positive correlation with FBG. Results of KEGG analysis and metagenomic sequencing showed that SL decoction could reduce the Prevotellaceae, Rikenellaceae, and Helicobacteraceae, which were related to lipopolysaccharide biosynthesis, riboflavin metabolism, and peroxisome, respectively. It could also upregulate the abundance of Bacteroidaceae, which contributed to the metabolism of starch and sucrose as well as pentoseglucuronate interconversions. In the species level, SL decoction significantly upregulates the relative abundance of Bacteroides_ acidifaciens which showed a significant negative correlation with FBG and was reported to be a potential agent for modulating metabolic disorders such as diabetes and obesity. In conclusion, SL decoction was effective in hypoglycemia and its mechanism may be related to regulating gut microbiota via upregulating Bacteroides_acidifaciens.

\section{Introduction}

Diabetes is a chronic glycemic metabolism disorder disease, which is associated with excessive calorie intake, lack of exer- cise, genetic predisposition, etc. According to the data from International Diabetes Federation (IDF), there are 463 million people that have been diagnosed with diabetes worldwide in 2019 and the number is expected to increase to 700 million 
by 2045 [1]. Along with the increasing prevalence of diabetes, its complications are also becoming big threats to human health. Increasing attention has been focused on diabetes. Mechanism researches have made great progress, and many effective drugs for diabetes have been developed by now. Except for modern medicines, Chinese herbal medicine has been proved to be a good alternative therapy for diabetes. For example, a study from Gao et al. demonstrated that TCM intervention could reduce the conversion rate of IGT to diabetes and improve insulin resistance [2]. Another randomized clinical trial showed that JinQi Jiangtang tablets (a Chinese patent medicine) could effectively reduce the incidence of diabetes mellitus and even reverse the prediabetes to normal $[3,4]$. Tianqi capsule, another Chinese patent medicine, also has been proved to decrease the incidence of T2DM in subjects with IGT. Therefore, traditional Chinese medicine is worthy to be further studied.

Shenlian decoction, which consists of Coptis and ginseng, is widely used in treating diabetes in China. Using Coptis and ginseng in treating diabetes can be traced back to the Tang dynasty, which was recorded in the book Qianjin fang, written by Sun Simiao. In the Ming dynasty, these two herbs were firstly used as a prescription and recorded as Shenlian decoction in the book Wanbinghuichun. Nowadays, many studies showed that both Coptis and ginseng have the function of mediating glucose metabolism [5-9]. Besides, a study from Yuan et al. demonstrated the function of Shenlian decoction in protecting pancreatic $\beta$ cell [10]. However, further studies are needed to clarify the mechanism of its antidiabetes function. In this study, we try to uncover the mechanism from the point of mediating the gut microbiota.

\section{Materials and Methods}

2.1. Drugs and Reagents. Coptis chinensis and Panax ginseng were purchased from the Beijing Kangmei Pharmaceutical Co. LTD, and the contents of representative chemical compositions were determined by the Research and Experimental Center of Beijing University of Chinese Medicine (Table 1). Metformin hydrochloride tablets were purchased from the Sino-American Shanghai Squibb Pharmaceuticals Ltd.

2.2. Animal Treatment and Fecal Sample Collection. All animal experiments were performed with the approval of the Ethics Committee for Experimental Animals of the Institute of Basic Theory for Chinese Medicine. Six-week-old male C57BL/KsJ$\mathrm{db} / \mathrm{db}$ mice and their normal littermates $(\mathrm{db} / \mathrm{m})$ were purchased from Changzhou Cavens Experimental Animal Co. LTD. (Approval Number: SCXK (su) 2016-0010). All animals were housed in an air-conditioned animal experiment room with a stable temperature of $22-24^{\circ} \mathrm{C}$, humidity of $50-60 \%$, a 12-hour light and dark cycle, and free access to water and food (standard chow diet). The $\mathrm{db} / \mathrm{db}$ mice with FBG levels above $13.9 \mathrm{mmol} / \mathrm{l}$ were randomly allocated into 5 groups $(n=8)$, including the diabetic control (DC) group (distilled water, $0.2 \mathrm{ml} / 10 \mathrm{~g}$ ), Huanglian (HL) group (Coptis chinensis, $4.55 \mathrm{~g} /$ $\mathrm{kg}$ ), Rensheng (RS) group (Panax ginseng, $0.455 \mathrm{~g} / \mathrm{kg}$ ), Shenlian (SL) group (Coptis chinensis and Panax ginseng, $4.55 \mathrm{~g}$ and $0.455 \mathrm{~g} / \mathrm{kg}$, respectively), and metformin (Met) group (metformin, $0.228 \mathrm{~g} / \mathrm{kg}$ ). The $\mathrm{db} / \mathrm{m}$ mice were used as normal control (NC group). Interventions were conducted by gavage $(0.2 \mathrm{ml} / 10 \mathrm{~g})$ for 8 weeks. Fasting blood glucose (FBG) was measured using a blood glucose meter (Johnson \& Johnson) in the tail vein blood once 4 weeks, for 8 weeks. After 8 weeks, fresh fecal samples from each animal were collected via a clean catch method, in which pellets are expressed directly into a sterile centrifuge tube and stored at $-80^{\circ} \mathrm{C}$. Fecal samples were sent to Shanghai Majorbio Bio-Pharm Technology Co., Ltd., for further processing.

2.3. Immunohistochemistry. The process of immunohistochemistry was conducted as previously [11]. The antibody of insulin was purchased from Proteintech (catalog: 15848-1-AP).

2.4. DNA Extraction, Library Construction, and Metagenomic Sequencing. Total genomic DNA was extracted from feces samples using the E.Z.N.A. ${ }^{\circledR}$ Soil DNA Kit (Omega Bio-Tek, Norcross, GA, U.S.) according to the manufacturer's instructions. The process of library construction and metagenomic sequencing was conducted as previously [12].

2.5. Sequence Quality Control and Genome Assembly. The data were analyzed on the free online platform of the Majorbio Cloud Platform (http://www.majorbio.com). The pairedend Illumina reads were trimmed for adaptors and lowquality reads (length $<50$ bp or with a quality value $<20$ or having $N$ bases) using fastp [13]. Reads were aligned to the mouse genome by BWA (http://bio-bwa.sourceforge.net, version $0.7 .9 \mathrm{a}$ ), and any hit associated with the reads and their mated reads were removed. Metagenomic data were assembled using MEGAHIT [14, 15] (https://github.com/ voutcn/megahit, version 1.1.2). Contigs with the length being or over $300 \mathrm{bp}$ were selected as the final assembling result, and then, the contigs were used for further gene prediction and annotation.

2.6. Species Annotation and Evaluation. Sample sequencing results were clustered. All sequences were divided into OTUs (Operational Taxonomic Units) according to different similarity levels using Uparse. The representative sequences of OTUs with their relative abundance were used to calculate community richness, community diversity, and community coverage index by mothur (version v.1.30.1). Then, the relative abundance table of representative sequences of OTUs was used for UniFrac principal coordinate analysis (PCoA) (Lozupone and Knight, 2005), species difference analysis, redundancy analysis, and correlation heatmap analysis.

2.7. Gene Prediction, Taxonomy, and Functional Annotation. Open reading frames (ORFs) from each assembled contig were predicted using MetaGene [16] (http://metagene.cb.k.u-tokyo .ac.jp/). The predicted ORFs (no less than $100 \mathrm{bp}$ ) were retrieved and translated into amino acid sequences with NCBI translation table. A nonredundant gene catalog was constructed by CD-HIT [17] (http://www.bioinformatics.org/cd-hit/, version 4.6 .1 ) with $90 \%$ sequence identity and $90 \%$ coverage. Reads after quality control were mapped to nonredundant gene catalog with 95\% identity using SOAP aligner [18] (http://soap .genomics.org.cn/, version 2.21), and gene abundance in each 
TABLE 1: Contents of the main identified compounds in SL extract.

\begin{tabular}{lcr}
\hline Herbs & Compounds & Contents \\
\hline & Berberine & $5.80 \%$ \\
Coptis chinensis & Epiberberine & $0.88 \%$ \\
& Coptisine & $1.62 \%$ \\
& Palmatine & $1.37 \%$ \\
\hline & Ginsenoside Rg1 & $0.24 \%$ \\
Panax ginseng & Ginsenoside Rb1 & $0.37 \%$ \\
& Ginsenoside Re & $0.27 \%$ \\
\hline
\end{tabular}

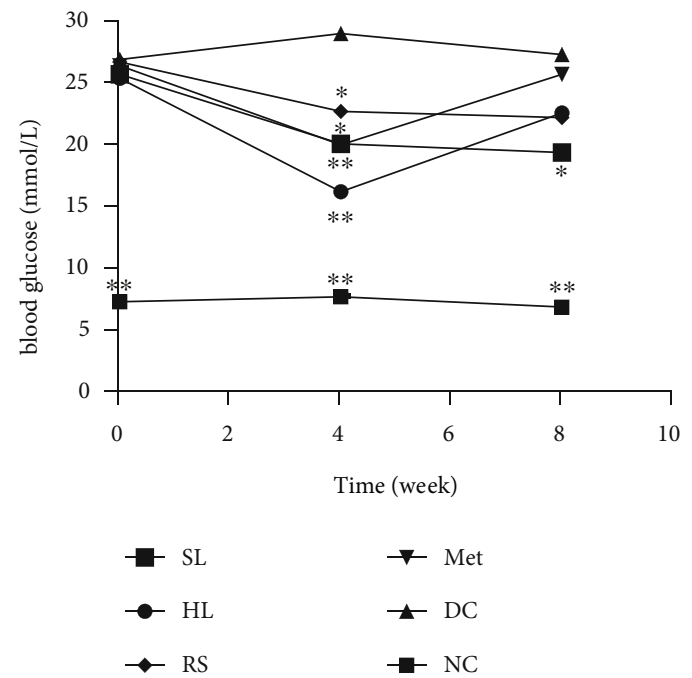

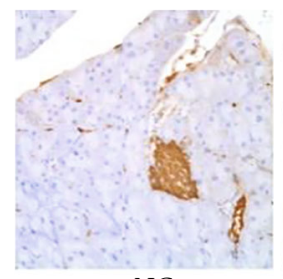

NC

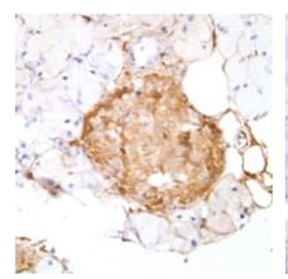

RS (a)

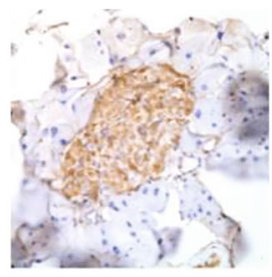

DC

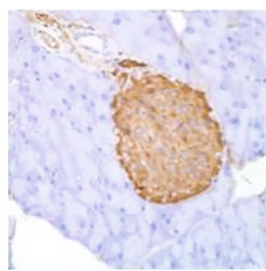

HL

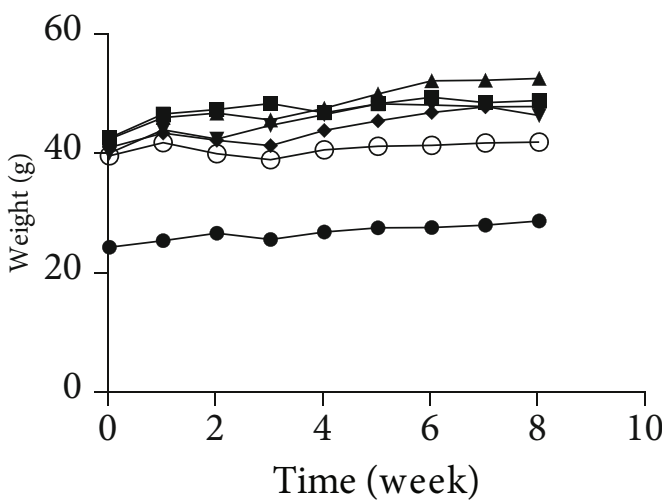

$\rightarrow \mathrm{NC}$

$\rightarrow \mathrm{RS}$

$-\mathrm{DC}$

$\rightarrow \mathrm{HL}$

$\triangle$ Met

(b)

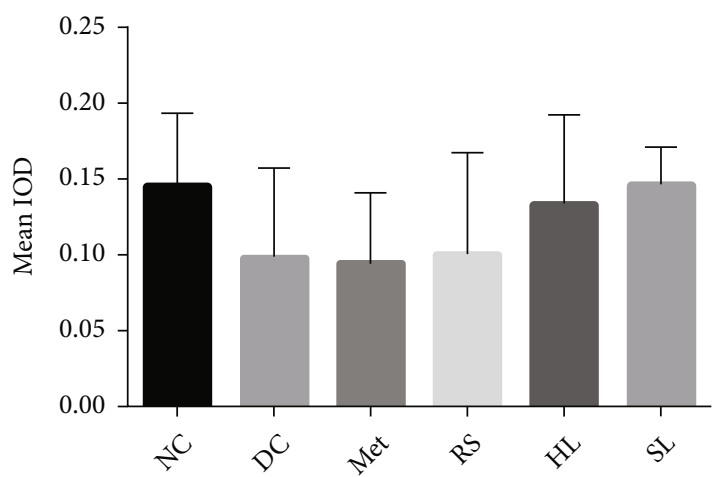

(d)

Figure 1: Effects on (a) FBG, (b) body weight, and (c, d) HOMA-IR. Immunohistochemistry of insulin in islet tissue. ${ }^{*} P<0.05$ vs. DC group; ${ }^{* *} P<0.01 ;{ }^{* * *} P<0.001$. 


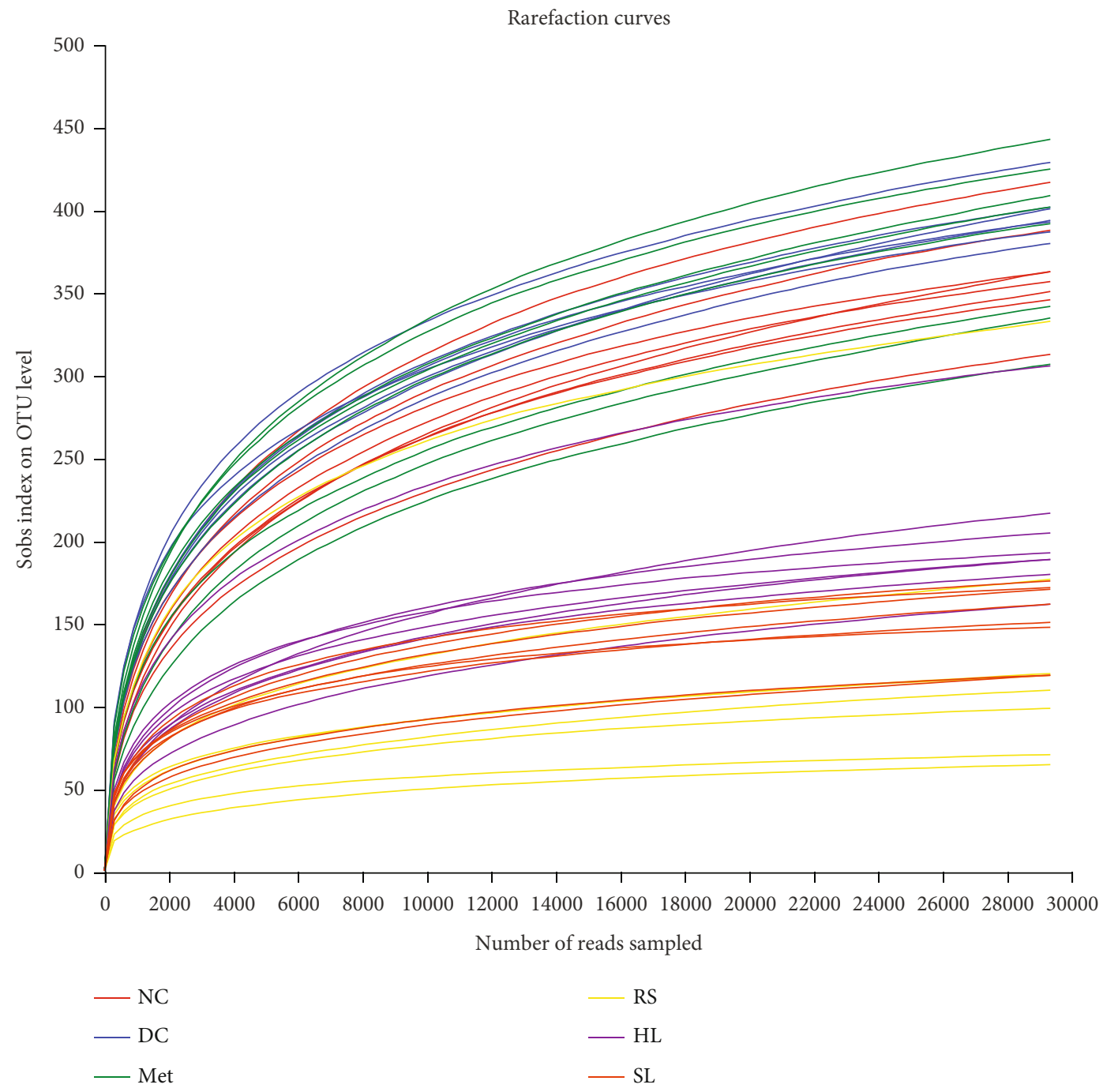

(a)

FIgUre 2: Continued. 


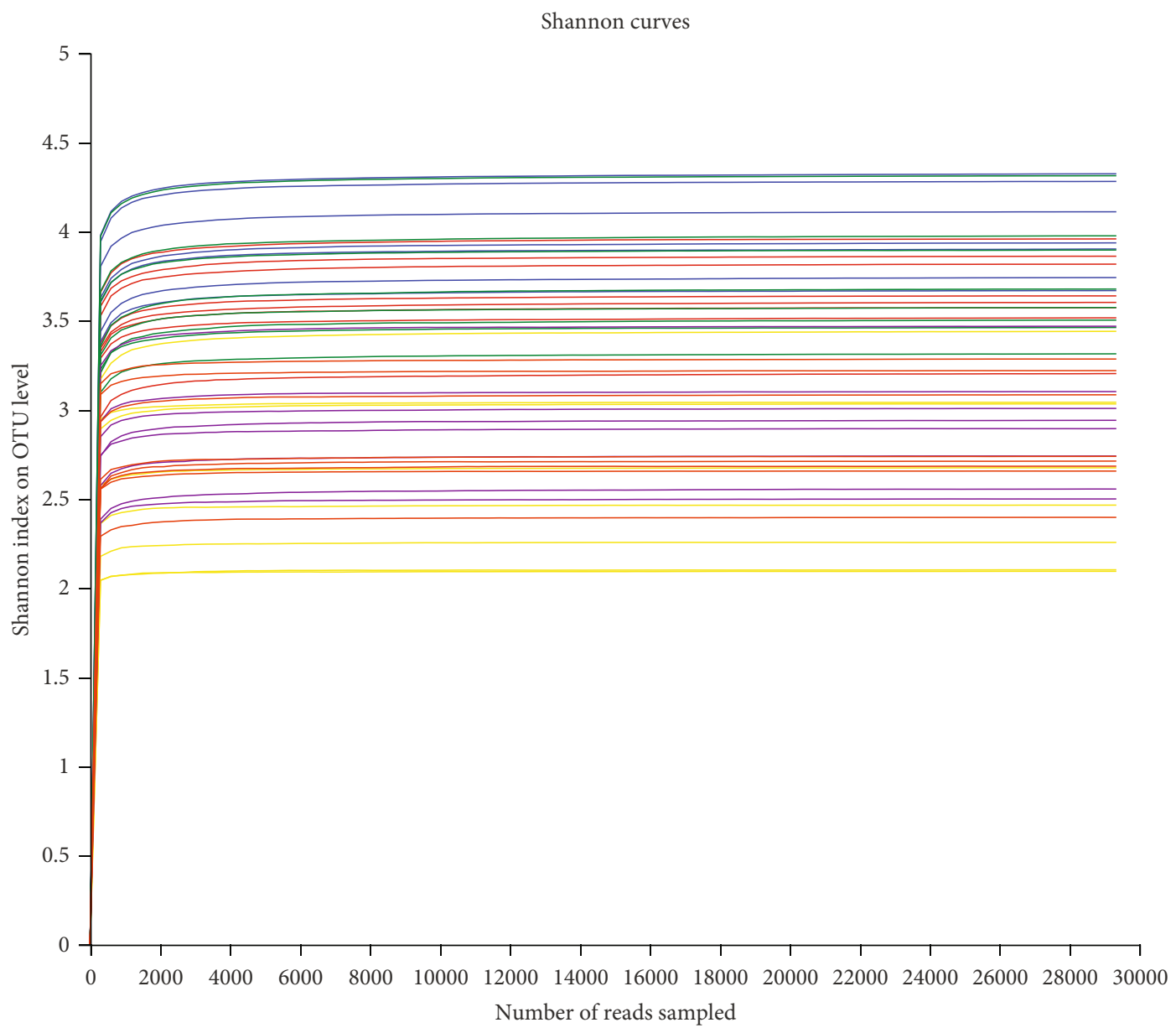

- NC

RS

- DC

$-\mathrm{HL}$

- Met

- SL

(b)

FIgUre 2: Continued. 
Coverage curves

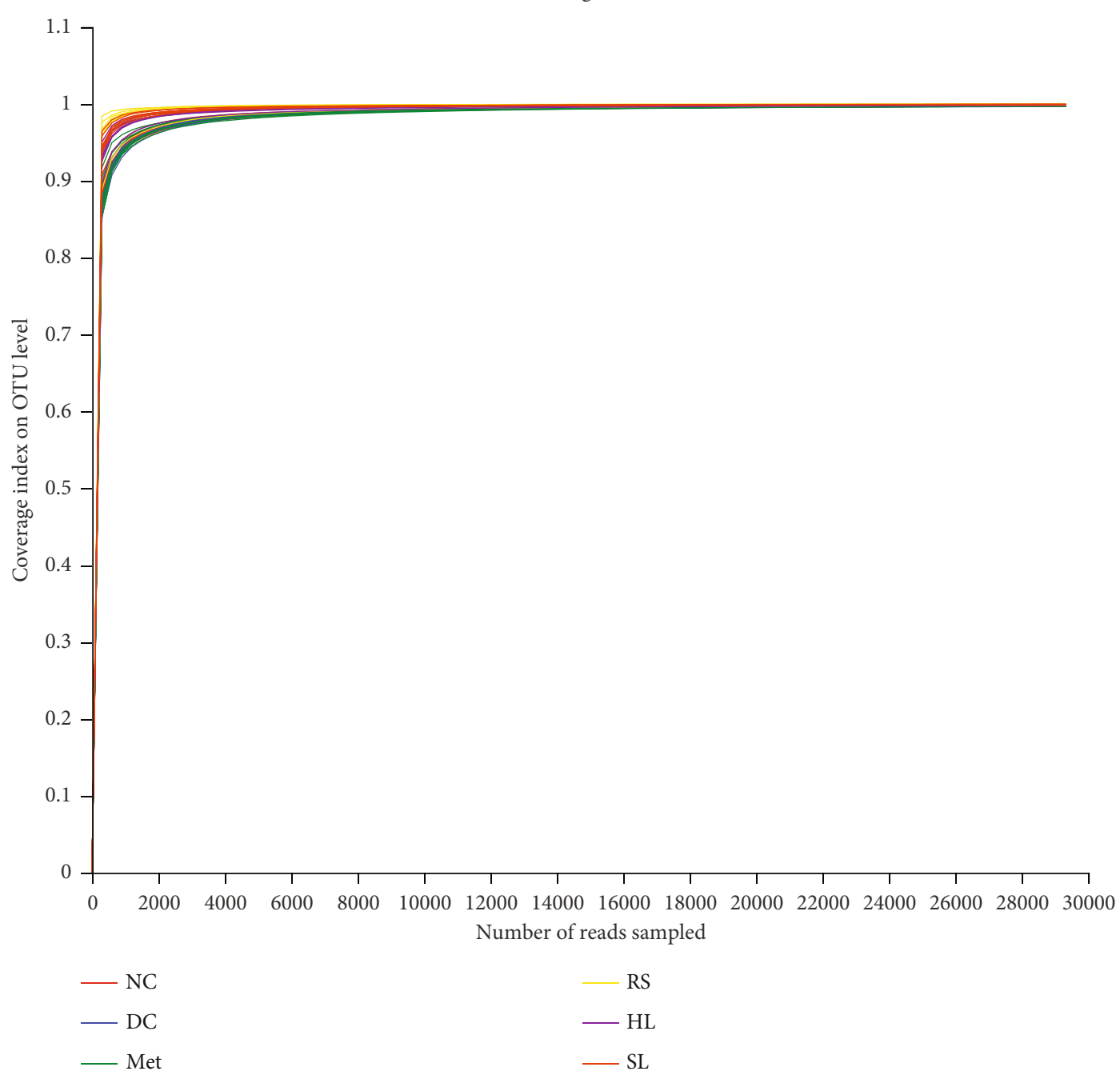

(c)

FIgUre 2: Continued. 


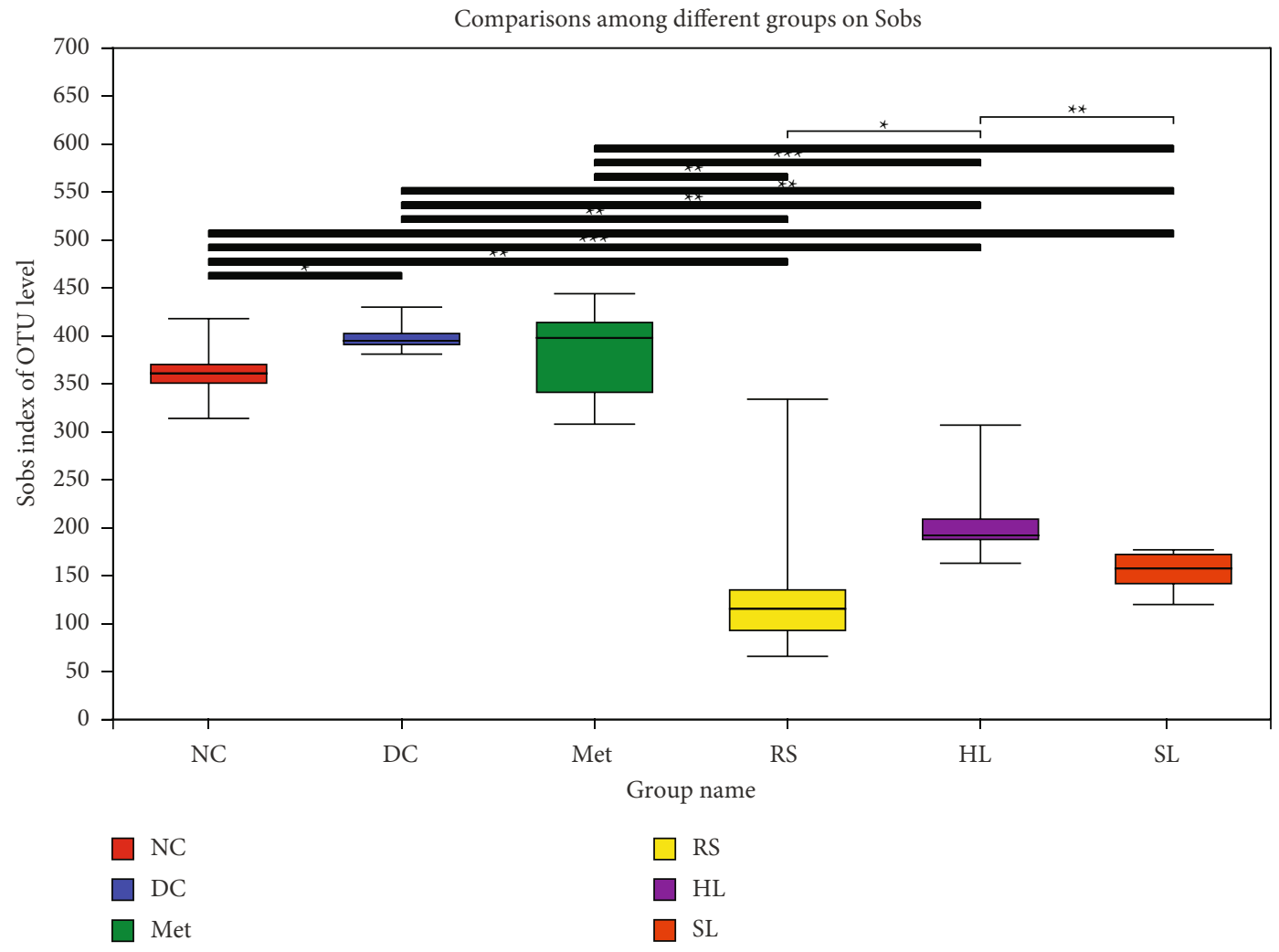

(d)

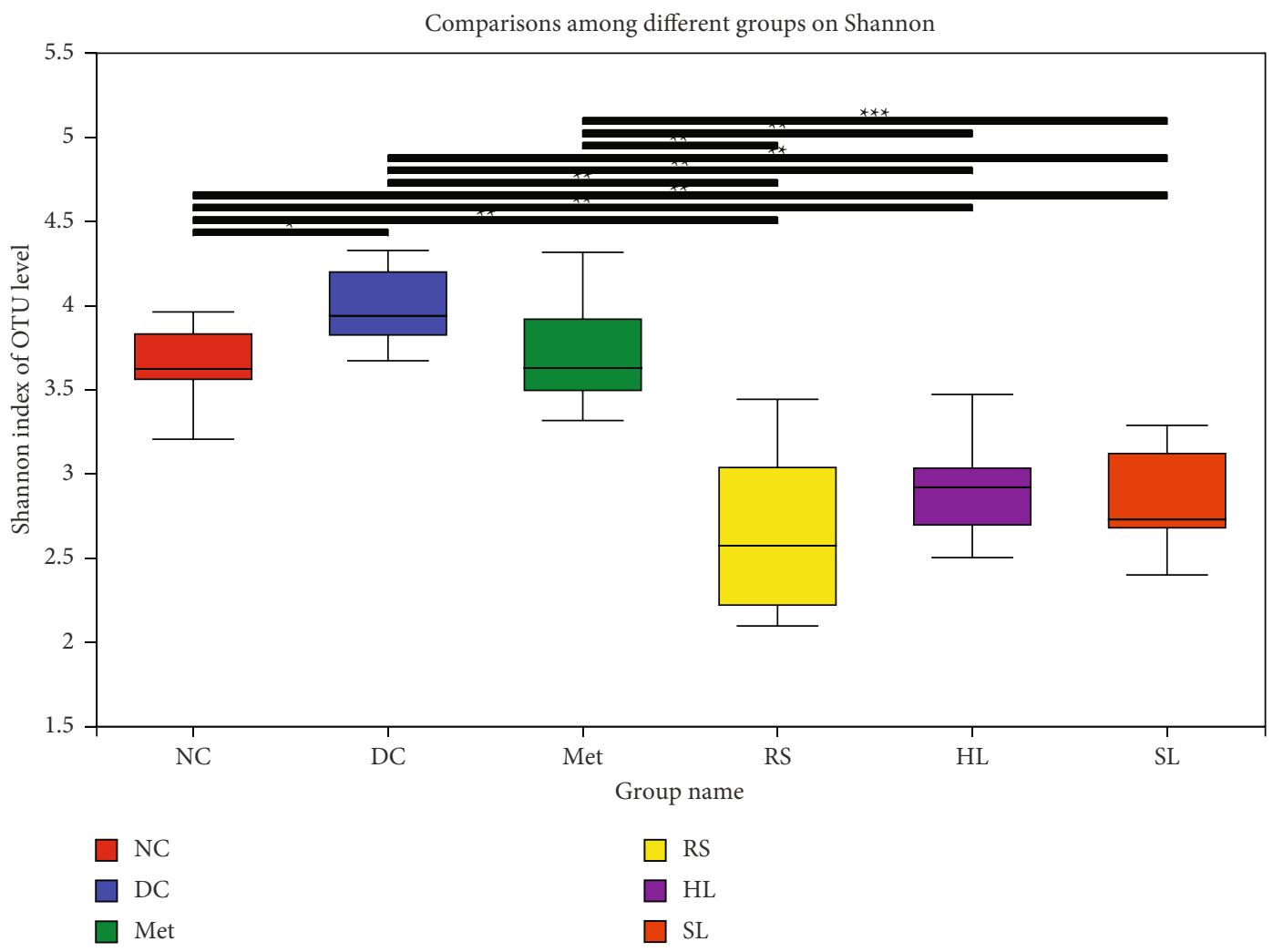

(e)

FIGURE 2: Alpha diversity analysis of bacterial relative abundances. (a) Sobs, (b) Shannon, and (c) coverage diversity curves of gut microbiota community, which reflect the richness, diversity, and coverage of the community, respectively. Comparisons among different groups on (d) Sobs and (e) Shannon of gut microbiota were significant. ${ }^{*} P<0.05$; ${ }^{* *} P<0.01$. 


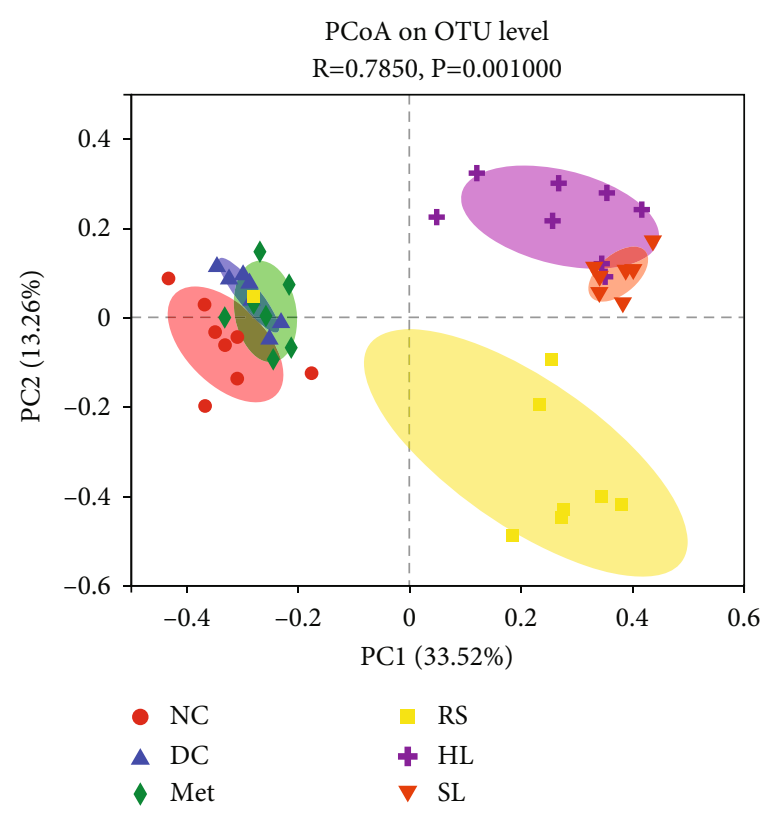

(a)

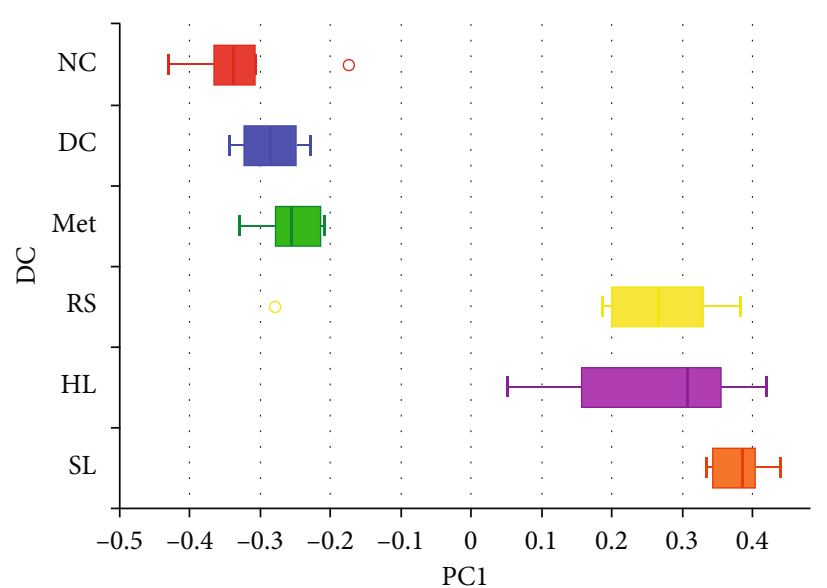

(b)

Difference analysis of gut microbiota on phylum level

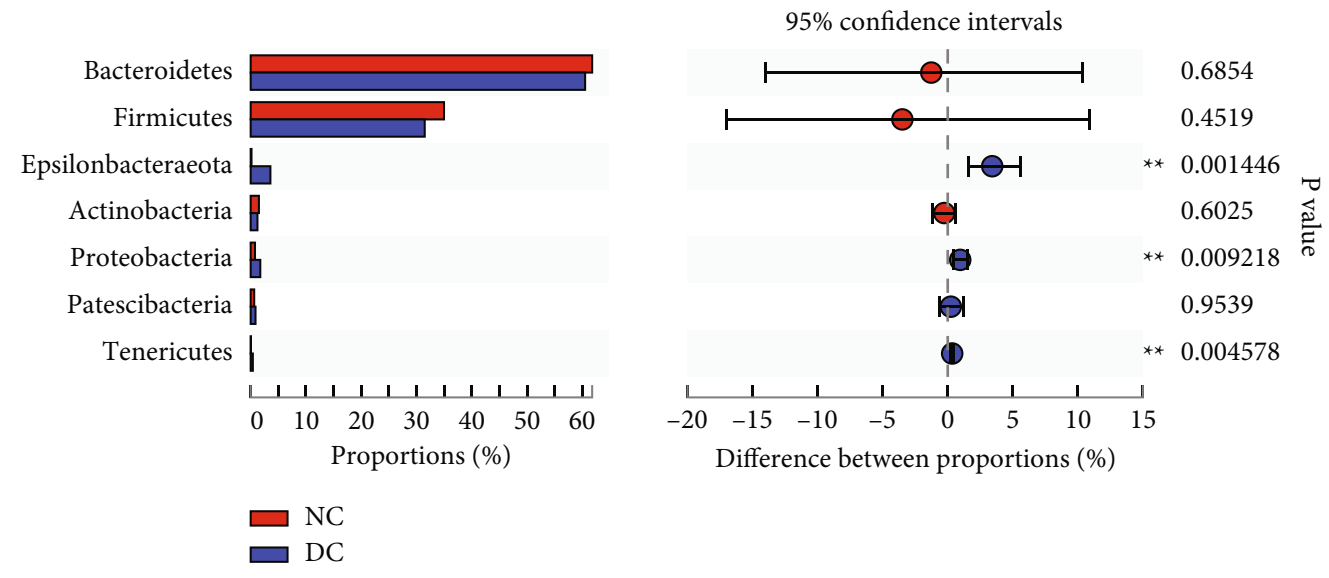

(c)

Difference analysis of gut microbiota on phylum level

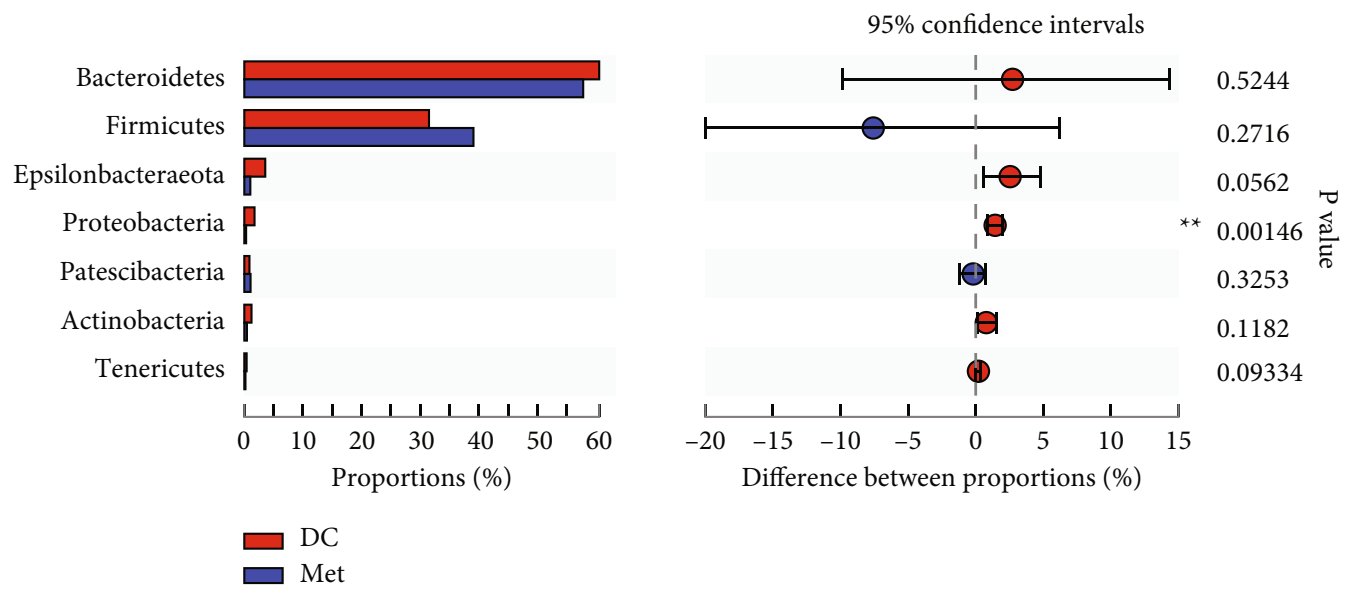

(d)

Figure 3: Continued. 


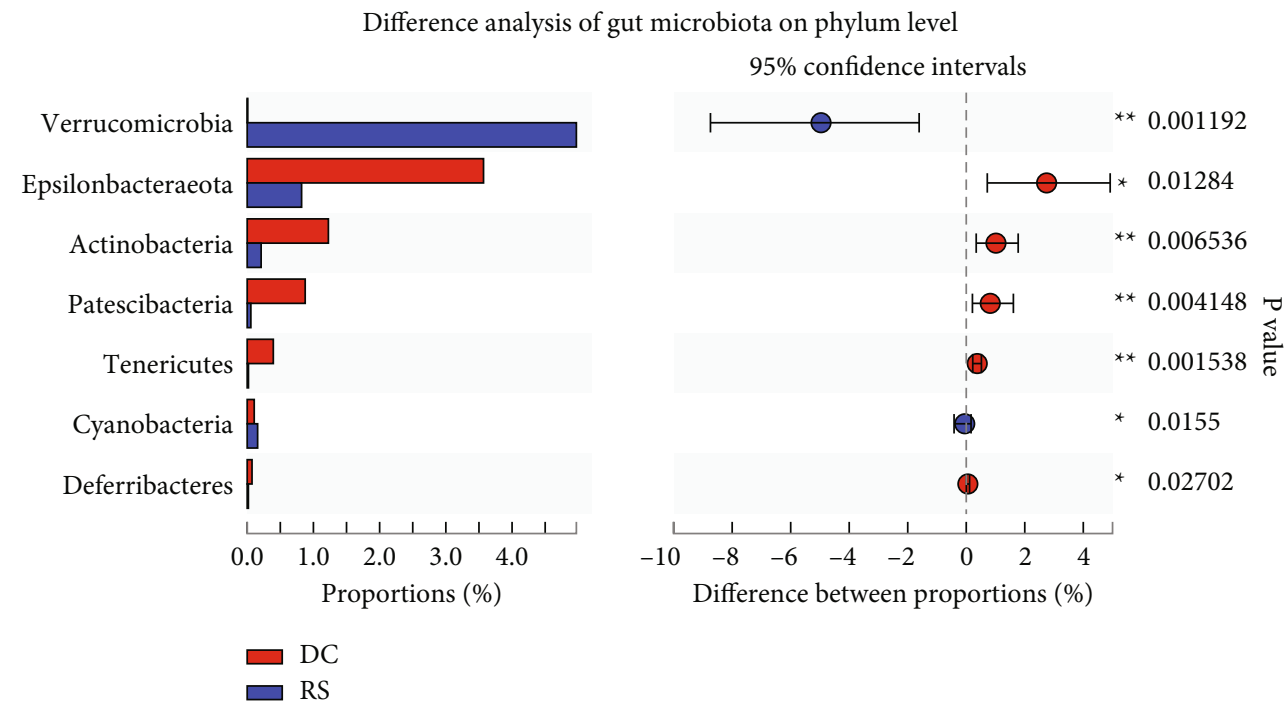

(e)

Difference analysis of gut microbiota on phylum level

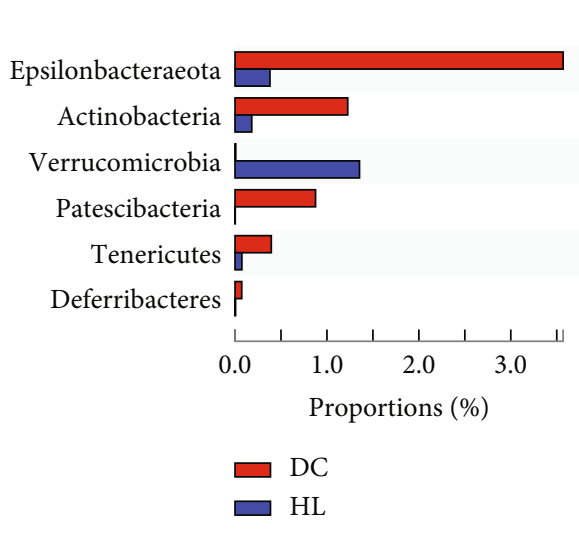

$95 \%$ confidence intervals

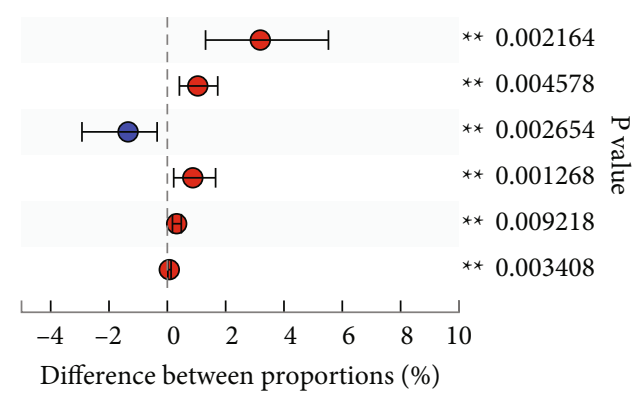

(f)

Difference analysis of gut microbiota on phylum level

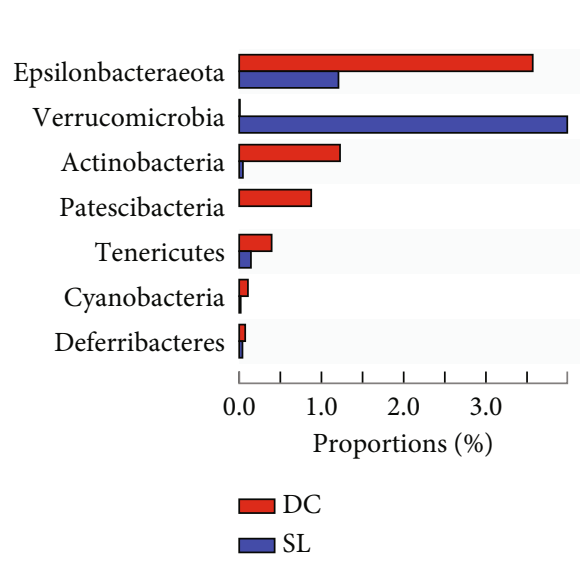

$95 \%$ confidence intervals

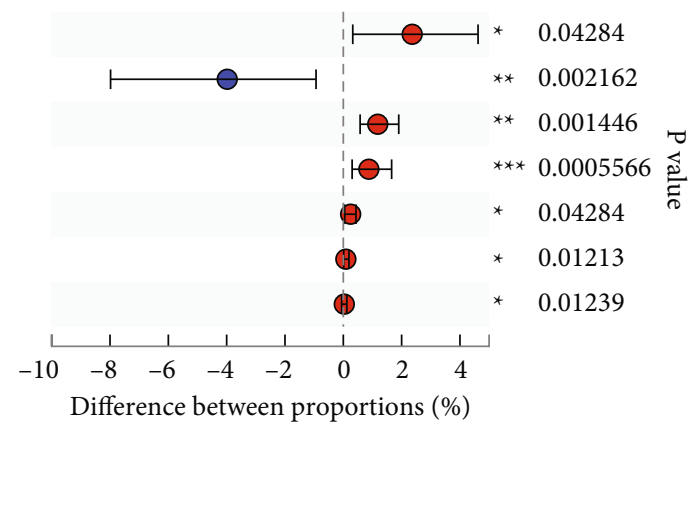

(g)

Figure 3: (a) Principal coordinate analysis (PCoA) of gut microbiota for mice. Weighted UniFrac PCoA plot and based on OTU abundance, each point represents the placenta microbiota of a sample, with main principal component (PC) scores: PC1 $=33.52 \%$ and PC2 $=13.26 \%$. (b) The boxplot represents the dispersion of distribution of different groups of samples on PC1. Relative abundance of phylum level was significantly different among (c) NC, (d) Met, (e) RS, (f) HL, (g) SL, and DC groups, ${ }^{*} P<0.05$, ${ }^{* *} P<0.01$, compared with the DC group. 
Difference analysis of gut microbiota on family level $95 \%$ confidence intervals
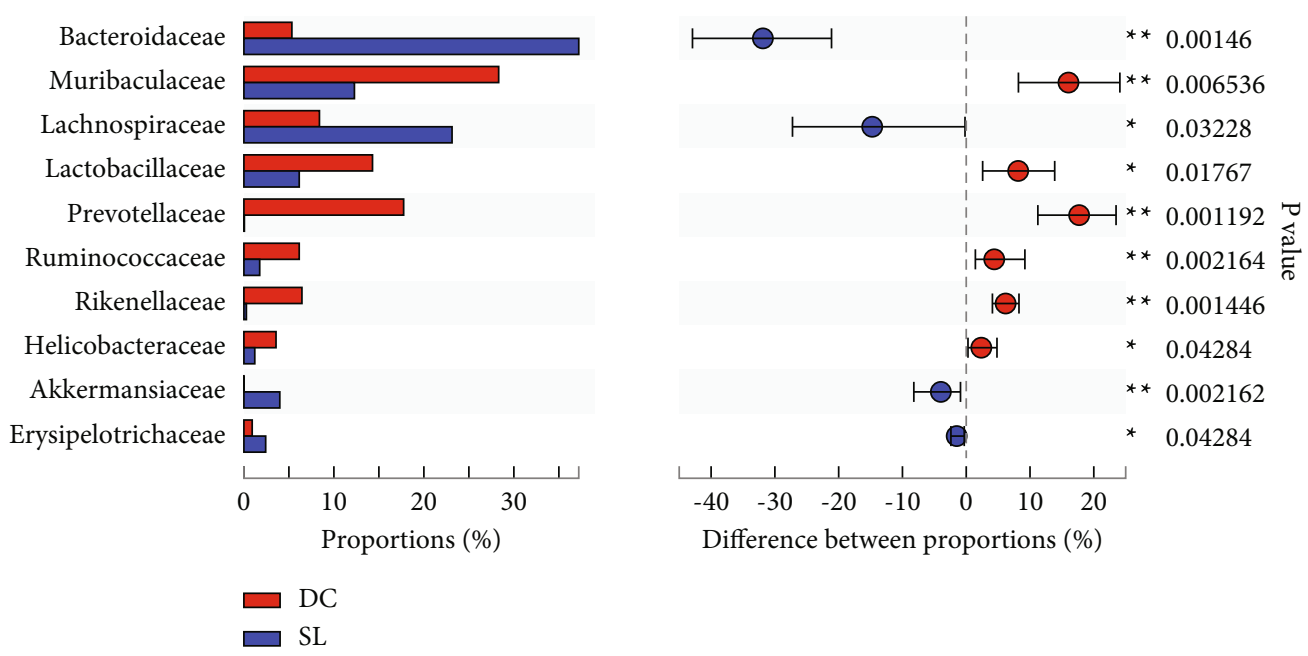

(a)

Difference analysis of gut microbiota on genus level

95\% confidence intervals

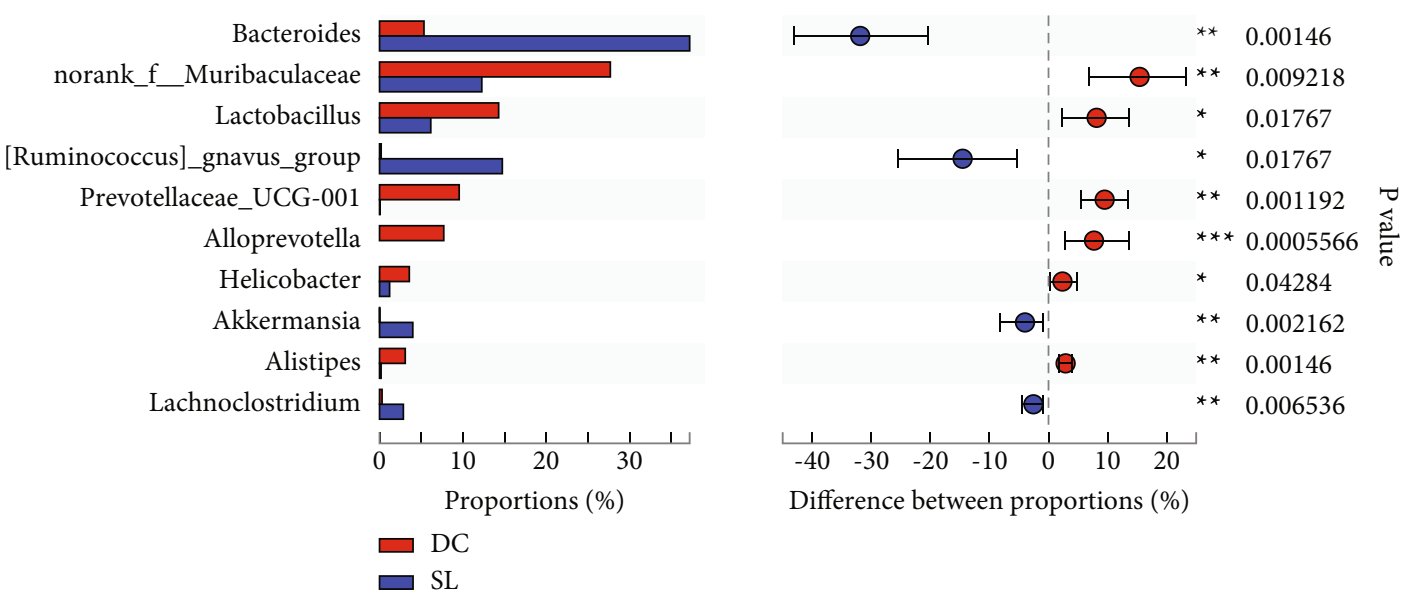

(b)

FIgURE 4: Continued. 
Difference analysis of gut microbiota on OTU level

95\% confidence intervals
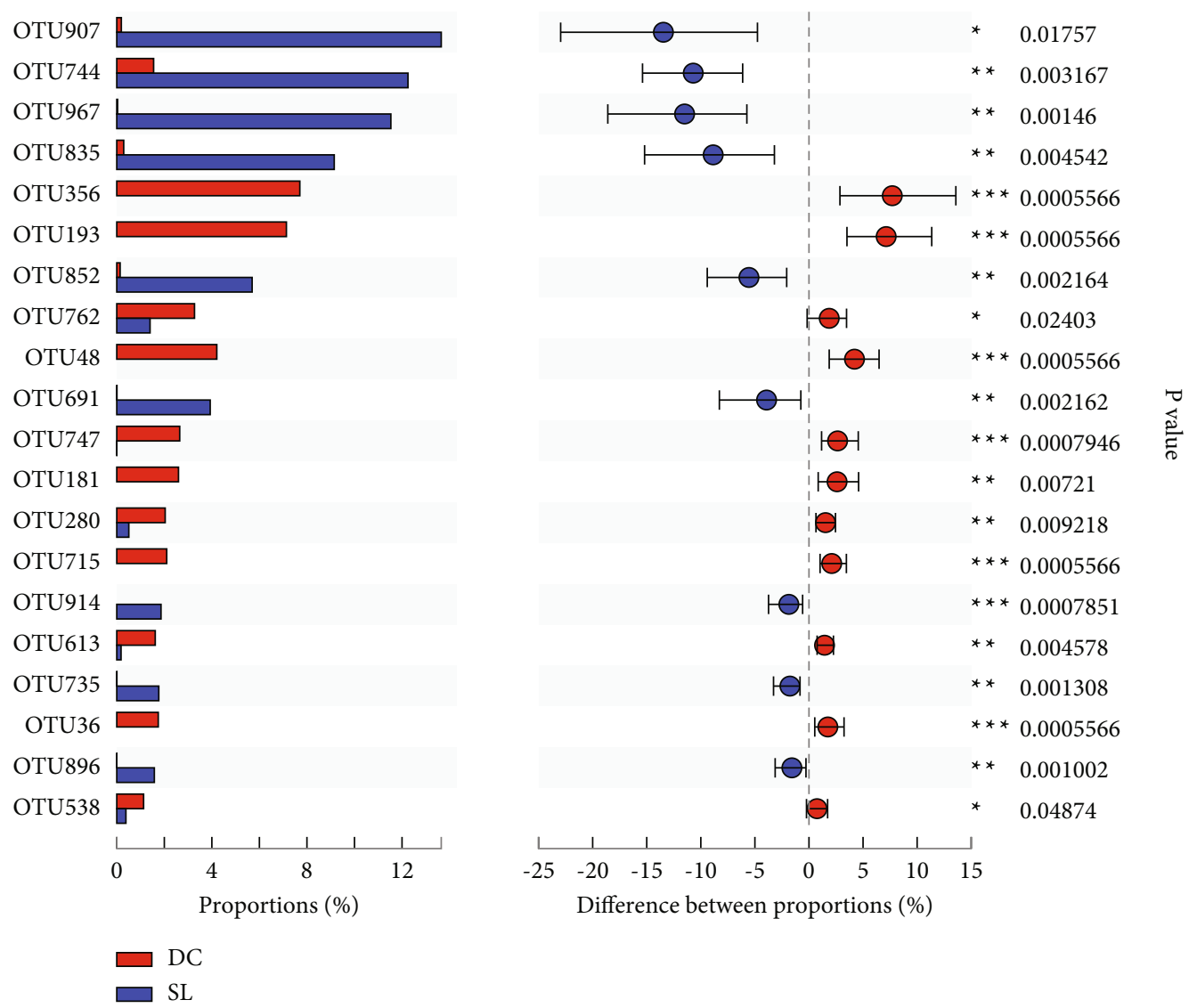

(c)

Figure 4: Continued. 


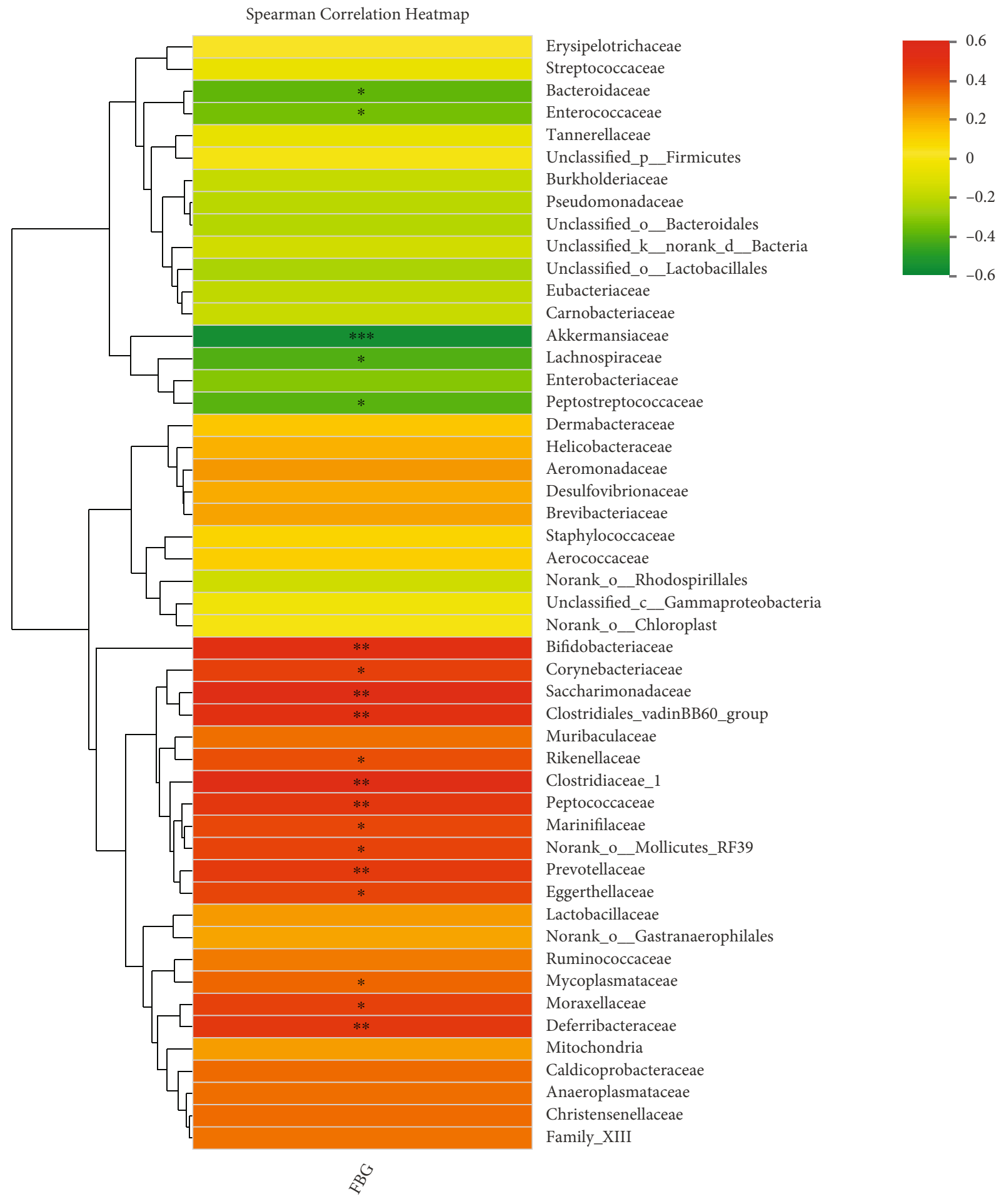

(d)

FIGURE 4: Significantly different between DC and SL groups of gut microbial community at the (a) family level, (b) genus level, and (c) OTU level. ${ }^{*} P<0.05$ and ${ }^{* *} P<0.01$, compared with the DC group. (d) Heatmap analysis indicating Spearman correlation between gut microbiota community and FBG on the family level, correlation $R$ values, and $P$ values were obtained by calculation; ${ }^{*} P<0.05$ and ${ }^{* *} P<0.01 ; R$ values were shown in different colors in the diagram. 


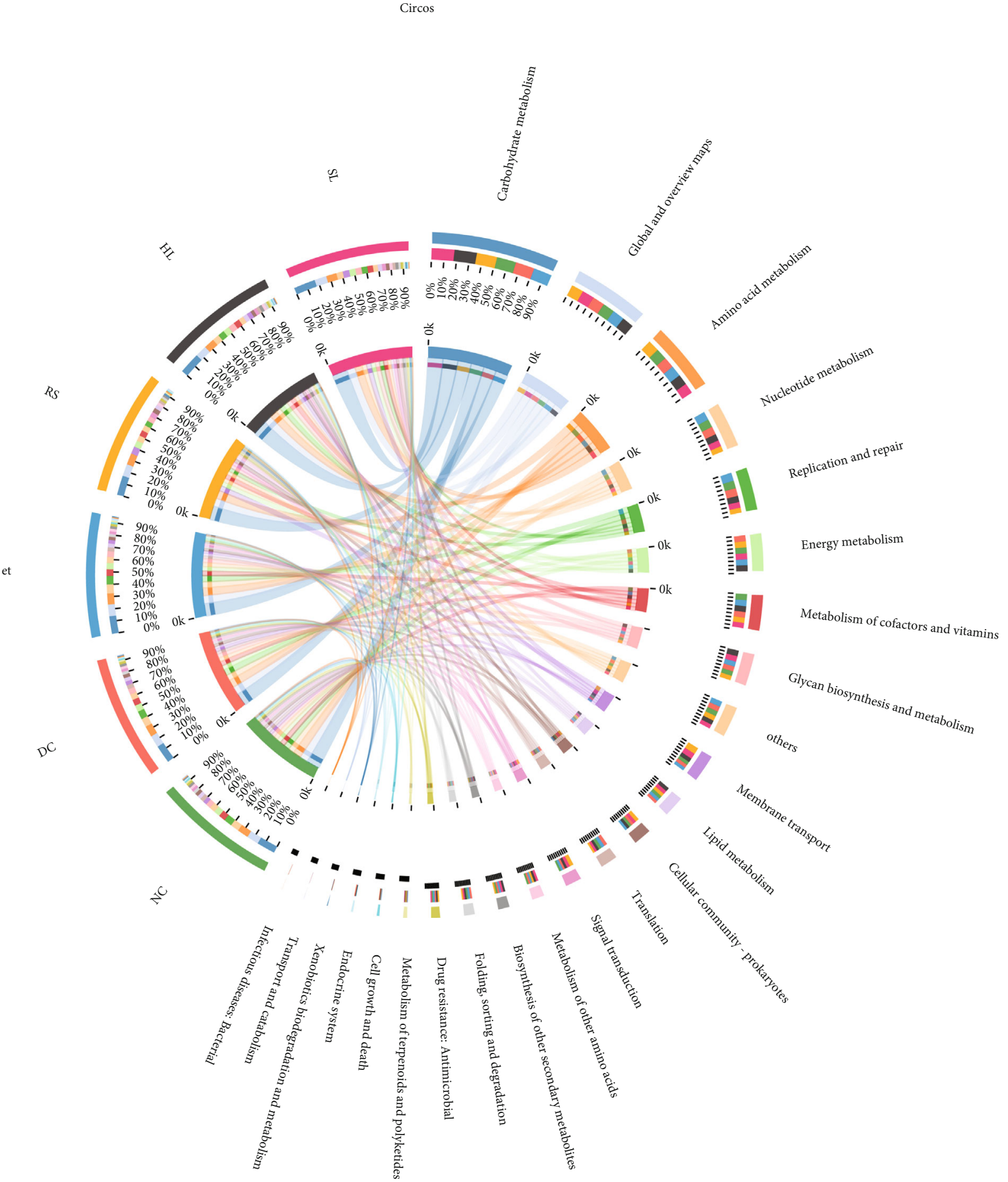

FIGURE 5: Circos diagram of the KEGG pathways enrichment analysis, the left half circle represents the function abundance composition of the group, and the right half circle represents the distribution ratio of the function.

sample was evaluated. Representative sequences of nonredundant gene catalog were aligned to NCBI NR database with $e$ value cutoff of $1 e-5$ using Diamond [19]. The KEGG annotation was conducted with Diamond (http://www.diamondsearch .org/index.php, version 0.8.35) against the Kyoto Encyclopedia of Genes and Genomes database (http://www.genome.jp/keeg/) with an $e$ value cutoff of $1 e-5$.
2.8. Statistical Analysis. The data are expressed as mean \pm SEM and analyzed using SPSS 22.0. Significant differences between the two groups were evaluated by Student's $t$-test or Welch's $t$ -test for samples that were normally distributed, and the Wilcoxon rank-sum test for samples that were not normally distributed. Significant differences among three or more groups were evaluated by one-way ANOVA with Bonferroni's multiple 


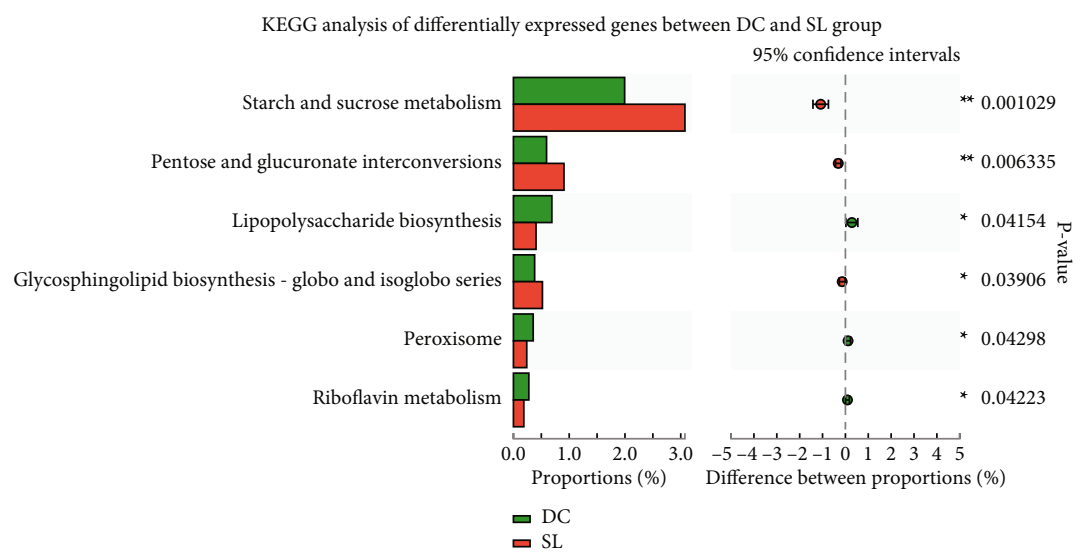

(a)

Correlation heatmap

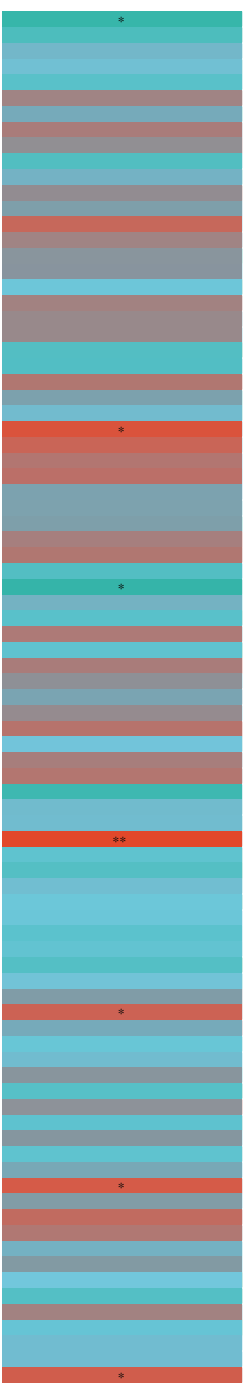

FBG

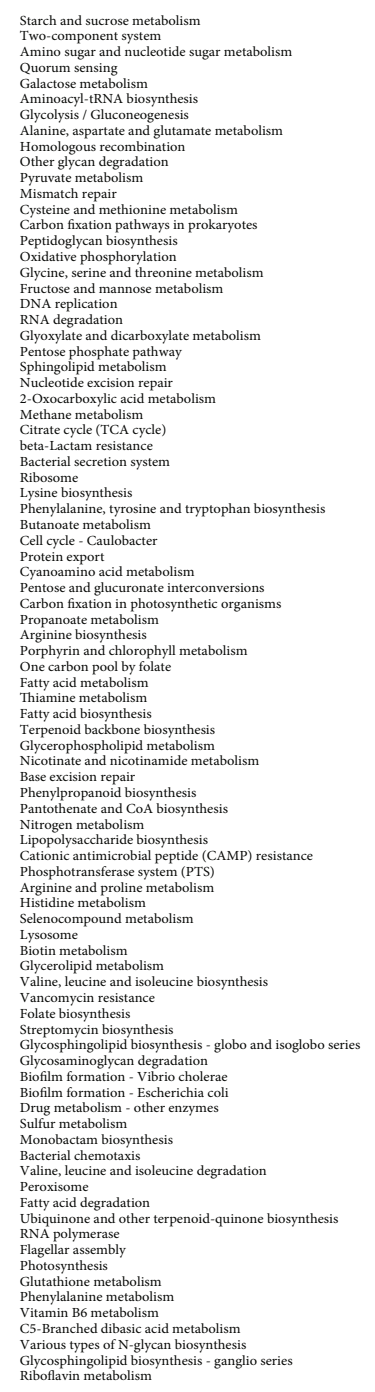

(b)

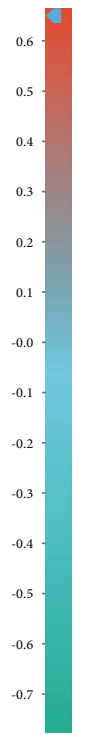

FIGURE 6: (a) KEGG analysis of differentially expressed genes between DC and SL groups. ${ }^{*} P<0.05$ and ${ }^{* *} P<0.01$, compared with the DC group. (b) Correlation heatmap between KEGG analysis and FBG in $\mathrm{db} / \mathrm{db}$ mice, the shades of color represent the richness of the function; correlation $R$ values and $P$ values were obtained by calculation; ${ }^{*} P<0.05$ and ${ }^{* *} P<0.01$; $R$ values were shown in different colors in the diagram. 
comparison test. The level of significance was set at $P<0.05$; ${ }^{*} P<0.05 ;{ }^{* *} P<0.01 ;{ }^{* * *} P<0.001$.

\section{Results}

3.1. Fasting Blood Glucose, Body Weight, and HOMA-IR Immunohistochemistry of $d b / d b$ Mice. FBG and body weight were evaluated after $6 \mathrm{~h}$ of food removal in the morning [20]. As shown in Figure 1(a), during 8 weeks of observation, $\mathrm{db} / \mathrm{db}$ mice presented significant hyperglycemia compared with the wild-type controls $(P<0.001)$. Metformin, HL, RS, and SL decoction significantly lowered blood glucose in the first 4 weeks $(P<0.01,0.05,0.01$, and 0.05$)$. However, in the eighth week, only the SL group had significantly lower blood glucose levels than the diabetic control group $(P<0.05)$. As for body weight, no significant difference was observed in the HL group, RS group, SL group, and Met group, compared with the DC group (Figure 1(b)). Immunohistochemistry of insulin in islet tissue was conducted to evaluate the function of $\beta$ cells. Results showed that less insulin was detected in $\mathrm{db} / \mathrm{db}$ mice compared with $\mathrm{db} / \mathrm{m}$ mice $(P=0.059)$, and HL $(P=0.278)$ and SL decoction $(P=0.053)$ could improve the insulin generation in islet tissue. The efficacy of SL decoction seems to be better than either RS or HL in promoting the insulin generation (Figures 1(c) and 1(d)).

3.2. Alpha Diversity Analysis of Bacterial Relative Abundances. To explore the effect of SL decoction on intestinal microbiota, gut microbiota of mice was analyzed by sequencing the gut bacterial 16S rRNA V3+V4 region and metagenomics. First, we used MiSeq run to analyze the structural changes of gut microbiota. In total, 2176480 usable raw sequences and 3222 OTUs were obtained, and a nonredundant catalog of 1609093 genes was constructed. Coverage (community coverage) diversity curves indicated that the sequencing results covered almost all the sequences in the sample, which means that the results could reflect the true situation of the microbiota community in the sample (Figures 2(a)-2(c)).

Sobs (the observed richness) and Shannon (the Shannon diversity index) diversity curves revealed that most of the diversity and richness of gut microbiota community had already been captured. The DC group had the significant higher microbiota diversity and richness compared with the NC group $(P<0.05)$. After 8 -week treatment, the increased microbiota diversity and richness were successfully reversed by HL, RS, and SL decoction (Figures 2(d) and 2(e)).

3.3. Composition Changes of Gut Microbiota. PCoA analysis showed a clear separation of the gut microbiota structure between DC and other groups (Figure 3(a)), especially when compared to the SL group with the DC group (Figure 3(b)). Further, the relative levels of gut microbiota at the phylum level were compared among groups. In the DC group, the abundances of Epsilonbacteraeota, Proteobacteria, and Tenericutes were significantly higher than the NC group (Figure 3(c)). Notably, HL, RS, and SL decoction could decrease the abundance of Epsilonbacteraeota and Tenericutes, and metformin decreased Proteobacteria abundance. What is more, HL, RS, and SL also increased the abundance of Verrucomicrobia and decreased the abundance of Actinobacteria, Patescibacteria, and Deferribacteres (Figures 3(d)-3(g)).

3.4. Correlation between Gut Microbiota Structure and FBG. SL decoction alters gut microbiota composition significantly in $\mathrm{db} / \mathrm{db}$ mice, the relative levels of gut microbiota were significantly different between DC and SL groups at the family level and genus level (Figures 4(a) and 4(b)), and top 20 OTU differences are shown in Figure 4(c). In order to identify specific gut bacteria that potentially are associated with FBG after SL treatment, Spearman's correlation analysis was performed between the gut microbiota community and FBG (Figure 4(d)). In total, 395 OTUs showed significant differences after SL treatment. 316 OTUs were decreased, and 79 were increased. Among the 79 OTUs enriched by SL, 37 OTUs showed a significant negative correlation with FBG (Supplementary Table 1). These OTUs belonged to Akkermansiaceae $(n=2)$, unclassified_o__Bacteroidales $(n=6)$, unclassified_k_norank_d_Bacteria $(n=1)$, Tannerellaceae $(n=2)$, Ruminococcaceae $(n=2)$, Rikenellaceae $(n=1)$, Peptostreptococcaceae $(n=1)$, norank_o__Rhodospirillales $(n=1)$, Muribaculaceae $(n=5)$, Lachnospiraceae $(n=8)$, Erysipelotrichaceae $(n=1), \quad$ Enterobacteriaceae $(n=1)$, and Bacteroidaceae $(n=6)$ on the family level. Among the 316 OTUs decreased by SL, 204 OTUs showed a significant positive correlation with FBG (Supplementary Table 2); these OTUs belonged to unclassified_o__Bacteroidales $(n=6)$, Tannerellaceae $(n=3)$, Streptococcaceae $(n=1$ ), Saccharimonadaceae $(n=1)$, Ruminococcaceae $(n=34)$, Rikenellaceae $(n=11)$, Prevotellaceae $(n=9)$, Peptococcaceae $(n=2)$, norank_o__Gastranaerophilales $(n=1)$, Muribaculaceae $(n=66)$, Moraxellaceae $(n=1)$, Marinifilaceae $(n=3)$, Lactobacillaceae $(n=2)$, Lachnospiraceae $(n=36)$, Helicobacteraceae $(n=1)$, Erysipelotrichaceae $(n=5)$, Eggerthellaceae $(n=7)$, Desulfovibrionaceae $(n=1)$, Deferribacteraceae $(n=1)$, Clostridiales_vadinBB60_group $(n=6$ ) , Clostridiaceae_1 $(n=1)$, Christensenellaceae $(n=2)$, Burkholderiaceae $(n=1)$, Bifidobacteriaceae $(n=1)$, and Bacteroidaceae $(n=2)$ on the family level.

3.5. Changes in Metabolic and Synthetic Functions of Gut Microbiota Caused by SL Decoction. To investigate the changes in metabolic and synthetic functions within the gut microbiota regulated by SL, the KEGG pathway enrichment analysis was performed. The most enriched functions included carbohydrate metabolism, amino acid metabolism, nucleotide metabolism, replication and repair energy metabolism, metabolism of cofactors and vitamins, and glycan biosynthesis (Figure 5). The KEGG analysis of differentially expressed genes between DC and SL is shown in Figure 6(a), and Spearman correlation between KEGG analysis and FBG is shown in Figure 6(b) on pathway level 3. The abundances of starch and sucrose metabolism, as well as pentose-glucuronate interconversions, showed a significant negative correlation with FBG and significantly increased after the SL treatment. In addition, the relative abundances of riboflavin metabolism, peroxisome, and lipopolysaccharide biosynthesis showed a significant positive correlation with FBG and significantly decreased after the SL treatment. 
Barplot of species and functional contribution analysis
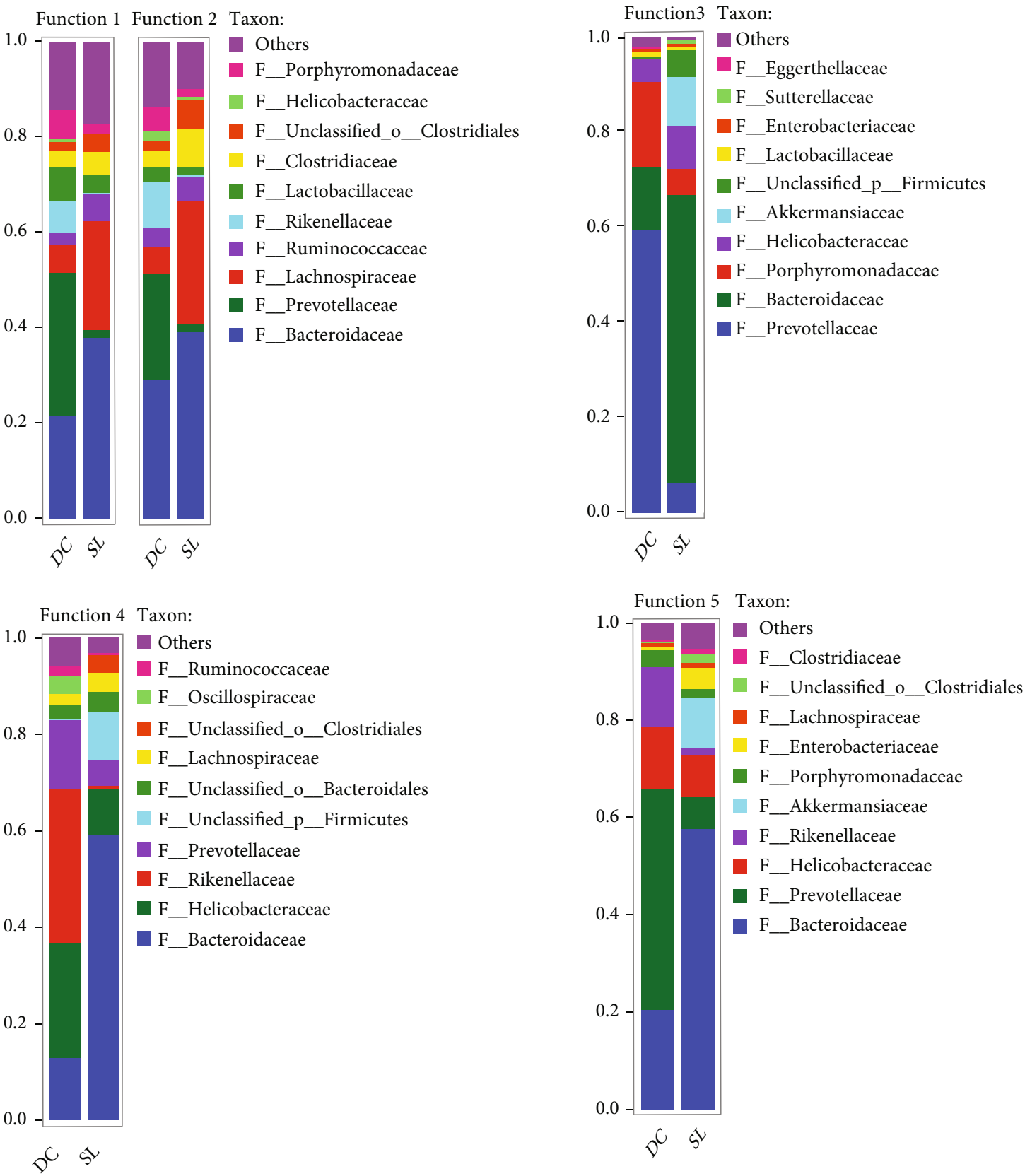

\author{
Function 1:starch and sucrose metabolism \\ Function 2:Pentose and glucuronate interconversions \\ Function 3:Riboflavin metabolism \\ Function 4:Peroxisome \\ Function 5:Lipopolysaccharide biosynthesis
}

FigURE 7: Barplot of species and functional contribution analysis indicates the dominant species composition of a particular function.

With the analysis of contribution capacity of different strains by metagenomic sequencing, Bacteroidaceae was found to display the greatest contribution to starch and sucrose metabolism and pentose and glucuronate interconversions. Prevotellaceae contributed the most to riboflavin metabolism and lipopolysaccharide biosynthesis. As for peroxisome, Rikenellaceae, Helicobacteraceae, and Prevotellaceae did a great contribution (Figure 7). Importantly, SL decoction could significantly decrease the abundance of Prevotellaceae, Rikenellaceae, and Helicobacteraceae and increase the abundance of Bacteroidaceae. The changes in relative abundance on Bacteroidaceae and Prevotellaceae were particularly significant in the SL group, when compared with other treatment groups (Figure 8). What is more, SL decoction could reduce the 


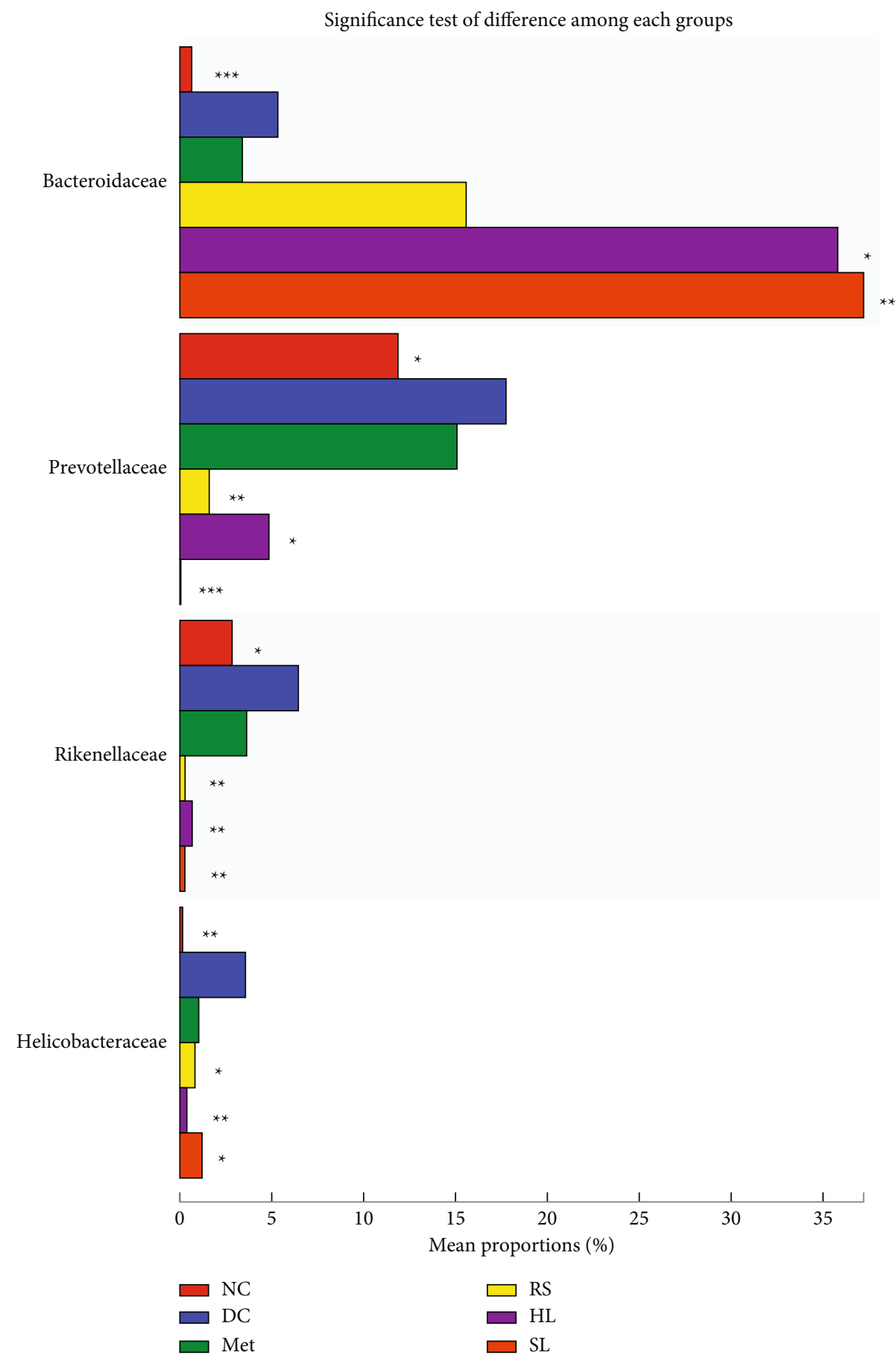

Figure 8: Significance test of difference of Bacteroidaceae, Prevotellaceae, Rikenellaceae, and Helicobacteraceae among each group, ${ }^{*} P<$ $0.05 ;{ }^{* *} P<0.01 ;{ }^{* *} P<0.001$, compared with the DC group.

relative abundance of 21 species positively correlated with FBG (Table 2) belonging to Prevotellaceae, Rikenellaceae, and Helicobacteraceae significantly, which were related to lipopolysaccharide biosynthesis, riboflavin metabolism, and peroxisome. It could also upregulate the relative abundance of 6 species negatively correlated with FBG (Table 3 ) belonging to
Bacteroidaceae, which contributed to the metabolism of starch and sucrose as well as pentose-glucuronate interconversions.

Data of DC and SL groups were shown as relative abundance (\%) of species and family in each group. Statistical analysis was performed by the Mann-Whitney $U$ test adjusted for multiple testing. 
TABLE 2: The relative abundance of 21 species reduced by SL.

\begin{tabular}{|c|c|c|c|c|}
\hline Family & Species & $\mathrm{DC}(\%)$ & SL (\%) & $P$ value \\
\hline Rikenellaceae & Uncultured_bacterium_g_Rikenellaceae_RC9_gut_group & 1.291 & 0.089 & 0.0022 \\
\hline Helicobacteraceae & Unclassified_g__Helicobacter & 2.647 & 0.002 & 0.0008 \\
\hline Prevotellaceae & Uncultured_Bacteroidales_bacterium_g_Prevotellaceae_UCG-001 & 2.101 & 0.000 & 0.0006 \\
\hline Prevotellaceae & Uncultured_Bacteroidales_bacterium_g_Prevotellaceae_UCG-001 & 0.177 & 0.000 & 0.0022 \\
\hline Prevotellaceae & Uncultured_Bacteroidales_bacterium_g_Prevotellaceae_UCG-001 & 0.128 & 0.000 & 0.0022 \\
\hline Rikenellaceae & Uncultured_bacterium_g__Rikenella & 0.321 & 0.000 & 0.0006 \\
\hline Rikenellaceae & Uncultured_bacterium_g__Alistipes & 0.131 & 0.002 & 0.0023 \\
\hline Rikenellaceae & Uncultured_bacterium_g__Alistipes & 0.224 & 0.003 & 0.0157 \\
\hline Prevotellaceae & Unclassified_f_Prevotellaceae & 0.013 & 0.000 & 0.0213 \\
\hline Rikenellaceae & Uncultured_bacterium_g__Alistipes & 0.304 & 0.000 & 0.0006 \\
\hline Rikenellaceae & Uncultured_bacterium_g__Alistipes & 0.475 & 0.000 & 0.0006 \\
\hline Prevotellaceae & Unclassified_f_Prevotellaceae & 0.009 & 0.000 & 0.0071 \\
\hline Rikenellaceae & Uncultured_bacterium_g__Alistipes & 0.064 & 0.000 & 0.0006 \\
\hline Rikenellaceae & Unclassified_g_Rikenellaceae_RC9_gut_group & 0.007 & 0.000 & 0.0019 \\
\hline Rikenellaceae & Unclassified_g_Rikenellaceae_RC9_gut_group & 1.745 & 0.000 & 0.0006 \\
\hline Prevotellaceae & Gut_metagenome_g_Alloprevotella & 7.701 & 0.000 & 0.0006 \\
\hline Rikenellaceae & Unclassified_g__Alistipes & 0.019 & 0.000 & 0.0071 \\
\hline Prevotellaceae & Uncultured_Bacteroidales_bacterium_g__Prevotellaceae_UCG-001 & 7.136 & 0.000 & 0.0006 \\
\hline Rikenellaceae & Unclassified_g__Alistipes & 1.329 & 0.000 & 0.0006 \\
\hline Prevotellaceae & Unclassified_f_Prevotellaceae & 0.006 & 0.000 & 0.0211 \\
\hline Prevotellaceae & Unclassified_f_Prevotellaceae & 0.255 & 0.000 & 0.0072 \\
\hline
\end{tabular}

TABLE 3: The relative abundance of 6 species increased by SL decoction.

\begin{tabular}{lcccc}
\hline Family & Species & DC (\%) & SL (\%) & $P$ value \\
\hline Bacteroidaceae & Unclassified_g_Bacteroides & 0 & 0.01576 & 0.002484 \\
Bacteroidaceae & Unclassified_g_Bacteroides & 0 & 0.009372 & 0.002537 \\
Bacteroidaceae & Unclassified_g_Bacteroides & 0 & 0.1793 & 0.00259 \\
Bacteroidaceae & Bacteroides_caecimuris & 0.4133 & 1.079 & 0.02403 \\
Bacteroidaceae & Unclassified_g_Bacteroides & 0.004382 & 0.9819 & 0.001321 \\
Bacteroidaceae & Bacteroides_acidifaciens & 1.55 & 12.25 & 0.003167 \\
\hline
\end{tabular}

\section{Discussion}

Diabetes is a chronic metabolic disease with high mortality and morbidity, which can lead to multiple organ injuries, including kidney, eyes, heart, nerve, and blood circulation.

Traditional Chinese medicine (TCM) has a long history of treating diabetes, which can trace back to the Han dynasty. During these thousands of years, ancient Chinese doctors accumulated lots of experience in treating diabetes. Shenlian decoction is one of them. An increasing number of studies showed that hypoglycemic Chinese medicines regulate glucose via mediating the gut microbiota. Quan et al. proved that ginseng extract could regulate the microbiota-lcFa-Bat axis, which played an important role in obesity [21]. Berberine, which is the main extract of Coptis chinensis, has been used in treating diabetes, and regulating gastrointestinal microbiota was proved to be the main mechanism of its antidiabetes function [22]. In this study, results demonstrated that Shenlian decoction had a good hypoglycemic function, which was better than either Coptis chinensis or ginseng after 8-week intervention. Results of insulin IHC also indicated that SL decoction had a better effect in protecting the function of $\beta$ cells than either ginseng or Coptis chinensis. Because in the theory of Chinese medicine, the basic mechanism of diabetes is "heat injures qi and yin." It means that pathogen heat, qi deficiency, and yin deficiency are main syndromes in diabetes patients. Therefore, in the clinic practice, TCM doctors always use Coptis chinensis to clear the heat and together with ginseng to tonify qi and yin. Some studies reported that ginseng failed to decrease blood glucose [23]. The reason is that ginseng can tonify qi and yin but cannot clear pathogen heat. So when using ginseng alone to treat diabetes, the efficacy cannot be good enough.

Increasingly, studies have shown that diabetes has a close relationship with the disorder of gut microbiota [24]. Dysbiosis of gut microbiota can disrupt the intestinal barrier, causing pathogens, proinflammatory cytokines, and harmful metabolites to enter the blood circulation, which contribute 
to the chronic low-grade inflammation [25]. Dysbiosis of gut microbiota in diabetes also manifests as a decline of shortchain fatty acid (SCFS) production. Low-grade SCFS induced decreased peptide YY and glucagon-like peptide(GLP-) 1 secretion, increased food intake, and disrupted intestinal barrier [26]. What is more, branched-chain amino acid (BCAA) biosynthesis is promoted when dysbiosis of gut microbiota happens and high BCAA level can cause insulin resistance $[27,28]$. In this study, results showed that SL decoction could alter gut microbiota composition in $\mathrm{db} / \mathrm{db}$ mice. Among the altered OTUs, 37 OTUs showed a significant negative correlation with FBG and 204 OTUs showed a significant positive correlation with FBG. What is more, as with the composition, the function of gut microbiota was also regulated by SL decoction, including starch and sucrose metabolism, pentose-glucuronate interconversions, riboflavin metabolism, peroxisome, and lipopolysaccharide biosynthesis, which were related to the FBS. Further analysis showed that SL decoction could decrease the relative abundance of Prevotellaceae, Rikenellaceae, and Helicobacteraceae and increase the abundance of Bacteroidaceae. These four families contributed mostly to FBS-related functions.

In the species level, SL decoction upregulates the relative abundance of Bacteroides_acidifaciens significantly, which showed a significant negative correlation with FBG and was reported to be a potential agent for modulating metabolic disorders such as diabetes and obesity. As reported, wild-type C57BL/6 mice fed with Bacteroides_acidifaciens were significantly more likely to gain less weight and fat and showed elevated insulin levels in serum, accompanied by increased serum glucagon-like peptide-1 and decreased intestinal dipeptidyl peptidase-4 [29]. Therefore, Bacteroides_acidifaciens upregulation may be the key mechanism for hypoglycemic function of SL decoction. Additionally, results of alpha diversity analysis showed that both HL and SL decoction reduced the diversity and richness of microbiota, which consisted with former studies [30,31]. This result might mainly be caused by the antibacterial effects of Coptis chinensis [32]. The reduced diversity and richness of gut microbiota in $\mathrm{db} / \mathrm{db}$ mice could give the beneficial bacteria more space to grow. It means that SL decoction regulates gut microbiota through a "restart microbiota" mode.

In conclusion, SL decoction was proved to be effective in hypoglycemia via regulating gut microbiota, especially the species of Bacteroides_acidifacien. In the future, a RCT should be conducted to prove its efficacy and further mechanism studies should focus on the families of Prevotellaceae, Rikenellaceae, Helicobacteraceae, and Bacteroidaceae.

\section{Data Availability}

The data sets used and/or analyzed during the current study are available from the corresponding author on reasonable request.

\section{Ethical Approval}

This study was carried out in accordance with the principles of the Basel Declaration and Recommendations of Animal Experimental Ethics. All animal experiments were performed with the approval of the Ethics Committee for Experimental Animals of Institute of Basic Theory for Chinese Medicine.

\section{Conflicts of Interest}

The authors declare that they have no conflicts of interest.

\section{Authors' Contributions}

Rui-xi Sun, Wei-jun Huang, and Yao Xiao participated in the design of this study, accomplishing animal tests and writing the dissertation. Dou-dou Wang and Guo-hua $\mathrm{Mu}$ helped to accomplish the animal experiments. He Nan, Bo-ran Ni, Xiao-qiang Huang, Xuan-quan Wang, and Yi-fan Liu helped to complete the preexperiment and data analysis. Jin-xi Zhao and Qiang-Fu designed the study and supervised all work. All authors approved the final manuscript. Rui-xi Sun, Weijun Huang, and Yao Xiao contributed equally to this work.

\section{Acknowledgments}

This work was supported by the National Natural Science Foundation of China (Grant nos. 81774272 and 81904135).

\section{Supplementary Materials}

Supplementary 1. Supplementary Table 1: species classification information of 37 OTUs.

Supplementary 2. Supplementary Table 2: species classification information of 204 OTUs.

\section{References}

[1] P. Saeedi, I. Petersohn, P. Salpea et al., "Global and regional diabetes prevalence estimates for 2019 and projections for 2030 and 2045: Results from the International Diabetes Federation Diabetes Atlas, $9^{\text {th }}$ edition," Diabetes Research and Clinical Practice, vol. 157, p. 107843, 2019.

[2] Y. Gao, H. Zhou, H. Zhao et al., "Clinical research of traditional Chinese medical intervention on impaired glucose tolerance," The American Journal of Chinese Medicine, vol. 41, no. 1, pp. 21-32, 2013.

[3] X. Sun, L. Guo, H. Shang et al., "The cost-effectiveness analysis of JinQi Jiangtang tablets for the treatment on prediabetes: a randomized, double-blind, placebo-controlled, multicenter design," Trials, vol. 16, no. 1, p. 496, 2015.

[4] H. Cao, M. Ren, L. Guo et al., "JinQi-Jiangtang tablet, a Chinese patent medicine, for pre-diabetes: a randomized controlled trial," Trials, vol. 11, no. 1, p. 27, 2010.

[5] B. Pang, L. H. Zhao, Q. Zhou et al., "Application of Berberine on Treating Type 2 Diabetes Mellitus," International Journal of Endocrinology, vol. 2015, Article ID 905749, 12 pages, 2015.

[6] W. Chen and P. Balan, "Review of ginseng anti-diabetic studies," Molecules, vol. 24, no. 24, p. 4501, 2019.

[7] J. Xie, "Ginseng and diabetes," The American Journal of Chinese Medicine, vol. 33, no. 3, pp. 397-404, 2005.

[8] H. Yuan, "Ginseng and diabetes: the evidences from in vitro, animal and human studies," Journal of Ginseng Research, vol. 36, no. 1, pp. 27-39, 2012. 
[9] S. Wang, Z. Xu, B. Cai, and Q. Chen, "Berberine as a potential multi-target agent for metabolic diseases: a review of investigations for berberine," Endocrine, Metabolic \& Immune Disorders Drug Targets, vol. 21, no. 6, pp. 971-979, 2021.

[10] F. Yuan, D. Wang, L. Ma et al., "Effects of Huanglian-RenshenDecoction, a Fixed Mixture of Traditional Chinese Medicine, on the Improvement of Glucose Metabolism by Maintenance of Pancreatic $\beta$ Cell Identity in $\mathrm{db} / \mathrm{db}$ Mice," Evidence-based Complementary and Alternative Medicine, vol. 2019, Article ID 1232913, 13 pages, 2019.

[11] W. J. Huang, Q. Fu, Y. H. Xiao et al., "Effect of Qufengtongluo Decoction on PI3K/Akt Signaling Pathway in the Kidney of Type 2 Diabetes Mellitus Rat (GK Rat) with Diabetic Nephropathy," Evidence-based Complementary and Alternative Medicine, vol. 2018, Article ID 8421979, 9 pages, 2018.

[12] D. Hu, J. Yang, Y. Qi, B. Li, K. Li, and K. M. Mok, "Metagenomic analysis of fecal archaea, bacteria, Eukaryota, and virus in Przewalski's horses following anthelmintic treatment," Frontiers in veterinary science, vol. 8, 2021.

[13] S. Chen, Y. Zhou, Y. Chen, and J. Gu, "fastp: an ultra-fast allin-one FASTQ preprocessor," Bioinformatics, vol. 34, no. 17, pp. i884-i890, 2018.

[14] H. Li and R. Durbin, "Fast and accurate short read alignment with Burrows-Wheeler transform," Bioinformatics, vol. 25, no. 14, pp. 1754-1760, 2009.

[15] D. Li, C. M. Liu, R. Luo, K. Sadakane, and T. W. Lam, "MEGAHIT: an ultra-fast single-node solution for large and complex metagenomics assembly via succinct de Bruijn graph," Bioinformatics, vol. 31, no. 10, pp. 1674-1676, 2015.

[16] N. HIDEKI, P. JUNGHO, and T. TOSHIHISA, "MetaGene: prokaryotic gene finding from environmental genome shotgun sequences," Nucleic Acids Research, vol. 34, no. 19, pp. 56235630, 2006.

[17] L. Fu, B. Niu, Z. Zhu, S. Wu, and W. Li, "CD-HIT: accelerated for clustering the next-generation sequencing data," Bioinformatics, vol. 28, no. 23, pp. 3150-3152, 2012.

[18] R. Li, Y. Li, K. Kristiansen, and J. Wang, "SOAP: short oligonucleotide alignment program," Bioinformatics, vol. 24, no. 5, pp. 713-714, 2008.

[19] D. H. Huson and B. Buchfink, "Fast and sensitive protein alignment using DIAMOND," Nature Methods, vol. 12, no. 1, pp. 59-60, 2015.

[20] J. Ayala, "Standard operating procedures for describing and performing metabolic tests of glucose homeostasis in mice," Disease Models \& Mechanisms, vol. 3, no. 9-10, pp. 525-534, 2010.

[21] L. H. Quan, C. Zhang, M. Dong et al., "Myristoleic acid produced by enterococci reduces obesity through brown adipose tissue activation," Gut, vol. 69, no. 7, pp. 1239-1247, 2020.

[22] L. Zhang, X. Wu, R. Yang et al., "Effects of berberine on the gastrointestinal microbiota," Frontiers in Cellular and Infection Microbiology, vol. 10, article 588517, 2021.

[23] D. Reeds, "Ginseng and ginsenoside Re do not improve $\beta$-cell function or insulin sensitivity in overweight and obese subjects with impaired glucose tolerance or diabetes," Diabetes Care, vol. 34, no. 5, pp. 1071-1076, 2011.

[24] Y. FAN, "Gut microbiota in human metabolic health and disease,” vol. 19, no. 1, pp. 55-71, 2021.

[25] G. MUSSO, R. GAMBINO, and M. CASSADER, "Obesity, diabetes, and gut microbiota," Diabetes Care, vol. 33, no. 10, pp. 2277-2284, 2010.
[26] Y. GHORBANI, K. J. P. SCHWENGER, and J. P. ALLARD, "Manipulation of intestinal microbiome as potential treatment for insulin resistance and type 2 diabetes," European Journal of Nutrition, vol. 60, no. 5, pp. 2361-2379, 2021.

[27] Z. ARANY and M. NEINAST, "Branched chain amino acids in metabolic disease," Current Diabetes Reports, vol. 18, no. 10, p. 76, 2018.

[28] H. Pedersen, "Human gut microbes impact host serum metabolome and insulin sensitivity," Nature, vol. 535, no. 7612, pp. 376-381, 2016.

[29] J. Yang, "Gut commensal_Bacteroides acidifaciens_ prevents obesity and improves insulin sensitivity in mice," Mucosal Immunology, vol. 10, no. 1, pp. 104-116, 2017.

[30] C. N. Li, X. Wang, L. Lei et al., "Berberine combined with stachyose induces better glycometabolism than berberine alone through modulating gut microbiota and fecal metabolomics in diabetic mice," Phytotherapy Research, vol. 34, no. 5, pp. 1166-1174, 2020.

[31] J. Garcia-Mazcorro, "Effect of barley supplementation on the fecal microbiota, caecal biochemistry, and key biomarkers of obesity and inflammation in obese $\mathrm{db} / \mathrm{db}$ mice," European Journal of Nutrition, vol. 57, no. 7, pp. 2513-2528, 2018.

[32] J. Li, D. Yan, L. Ma et al., "A quality evaluation strategy for Rhizoma coptidis from a variety of different sources using chromatographic fingerprinting combined with biological fingerprinting," Chinese Science Bulletin, vol. 58, no. 33, pp. 4092-4100, 2013. 